\title{
Article \\ Emerging Applications of Bio-Inspired Algorithms in Image Segmentation
}

\author{
Souad Larabi-Marie-Sainte ${ }^{*}+\left(\mathbb{\infty}\right.$, Reham Alskireen $^{+}(\mathbb{1})$ and Sawsan Alhalawani ${ }^{+}(\mathbb{C}$ \\ Computer Science Department, College of Computer and Information Sciences, Prince Sultan University, \\ Riyadh 11586, Saudi Arabia; rskireen@psu.edu.sa (R.A.); shalawani@psu.edu.sa (S.A.) \\ * Correspondence: slarabi@psu.edu.sa \\ t These authors contributed equally to this work.
}

Citation: Larabi-Marie-Sainte, S. Alskireen, R.; Alhalawani, S. Emerging Applications of Bio-Inspired Algorithms in Image Segmentation. Electronics 2021, 10, 3116. https://doi.org/10.3390/ electronics10243116

Academic Editor: Ying Tan

Received: 31 October 2021

Accepted: 6 December 2021

Published: 14 December 2021

Publisher's Note: MDPI stays neutral with regard to jurisdictional claims in published maps and institutional affiliations.

Copyright: (c) 2021 by the authors. Licensee MDPI, Basel, Switzerland. This article is an open access article distributed under the terms and conditions of the Creative Commons Attribution (CC BY) license (https:/ / creativecommons.org/licenses/by/ $4.0 /)$.

\begin{abstract}
Image processing is one example of digital media. It consists of a set of operations to handle an image. Image segmentation is among its main important operations. It involves dividing the image into several parts or regions to extract vital information or identify relevant objects. Many techniques of artificial intelligence, including bio-inspired algorithms, have been used in this regard. This article collected the state-of-the-art studies presenting image-segmentation techniques combined with four bio-inspired algorithms including particle swarm optimization (PSO), genetic algorithms (GA), ant colony optimization (ACO), and artificial bee colonies (ABC). This research work aimed at showing the importance of image segmentation and its combination with these algorithms. This article provides insights on how these algorithms are adapted to image-segmentation combinatorial problems, which assist researchers to start the first hands-on application. It also discusses their setting parameters and the highly used algorithms such as PSO, GA, ACO, and ABC. The article presents new research directions in image segmentation based on bio-inspired algorithms.
\end{abstract}

Keywords: genetic algorithms; particle swarm optimization; ant colony optimization; bee colony optimization; image processing; image segmentation

\section{Introduction}

Image processing is a collection of operations applied to an image for improvement or extraction of some interesting information. It is one of the fast and rising technologies. Image watermarking, image segmentation, image filtering, and image classification are the main operations performed in this field. Therefore, these operations require continual improvement. This article aimed at reviewing image segmentation using artificial intelligence.

Nowadays, artificial intelligence (AI) is an emerging field that aims to handle the imitation of human intelligence to computers. AI techniques are considered as crucial in technology, contributing in looking for solutions to many challenging problems that different applications in computer science face (for example, in medicine [1]). Bio-inspired algorithms are well-known techniques of AI in solving difficult and combinatorial optimization problems. They are population-based techniques stimulated by behavior in animals.

Bio-inspired algorithms are capable to escape the local optimum and find the global optimum due to their exploration and exploitation aspects. The bio-inspired algorithms are combined with image-segmentation techniques with the aim to find the optimal parameters required in the segmentation techniques such as finding the centers in the Cmeans-clustering segmentation technique or determining the number of thresholds in the thresholding segmentation. Finding these parameters is a complex problem that requires robust optimization techniques such as the bio-inspired algorithms.

In this review, four well-known bio-inspired computing techniques are presented, including PSO [2], GA [3], ABC [4], and ACO [5]. 
Several Image Segmentation surveys have been published. For example, Khan and Ravi [6] along with Sonawane and Dhawale [7] introduced the six well-known imagesegmentation approaches including partial differential equations (PDEs), artificial neural network (ANNs), the edge approach, the threshold approach, the fuzzy theory approach, and the region approach. Liang et al. [8] reviewed image-segmentation techniques based on evolutionary algorithms such that GA, genetic programming, differential equation, and PSO. Zaitoun et al. [9] presented different segmentation techniques related to layerObased segmentation and block-based segmentation. Yuheng et al. [10] and Chauhan et al. [11] addressed four image-segmentation techniques cited above and discussed the advantages and the disadvantages of each approach. Chouhan et al. [12] and Chouhan et al. [13] surveyed three main techniques of AI used in segmentation such that the genetic algorithms, fuzzy logic, and ANN. However, the existing surveys addressed the classical image-segmentation approaches, and some of them were combined with AI. Only three works $([8,12,13])$ surveyed the evolutionary algorithms-based image segmentation, focusing on GA and PSO.

This review selects four well-known evolutionary algorithms and discusses the stateof-the-art-related image segmentation. These algorithms are mainly used in this field and yielded good results. The surveyed studies were published between 2003 and 2021, but only the studies published in the last six years were described in detail. This study aimed at assisting the researchers to achieve their work in this domain. The contribution of this review article consists of:

1. Presenting four bio-inspired-algorithm-based image-segmentation techniques not provided in previous surveys.

2. Indicating the well-used AI algorithms in solving image segmentation to help researchers choose the best segmentation technique.

3. Emphasizing the algorithms that are not mainly used in this regard to open new opportunities in handling and enhancing image segmentation.

4. Presenting various databases dedicated for the use in image segmentation to be served as a repository for future research.

5. Investigating the parameter setting and recommending the most-used values for the non-expert AI researchers working in image segmentation.

6. Suggesting new directions and future works for exploration to expand this research field.

The article is organized as follows. Section 2 presents image processing and image segmentation. Section 3 addresses the four bio-inspired algorithms including GA, PSO, $\mathrm{ACO}$, and $\mathrm{ABC}$. Section 4 displays the main state-of-the-art image-segmentation studies, based on the four bio-inspired algorithms. Section 5 presents a deep and detailed discussion about the surveyed research works. Section 6 suggests new directions and concludes this study.

\section{Image Segmentation}

Image processing aims at extracting valuable information from an image by carrying out a set of signal-processing methods, which is usually considered as two-dimensional signals. This is performed through two types of methods including analog- and digitalimage processing. This study focused on the digital-image-processing techniques that involve manipulating images by using computers.

There are many useful applications of image processing such as image sharpening, blurring, gray scale conversion, and edges detection that can be utilized in different domains such as pattern recognition, objects detection, and medical-field applications.

Image segmentation aims to split an image into multiple areas or a set of pixels in order to extract relevant information or identify objects in digital images. Image segmentation results in a collection of contours taken from the image or a collection of sections that construct together the whole image. Image segmentation is required in different vital applications including medical imaging, retrieval of image content, object detection, and face detection/recognition. 
Image-segmentation algorithms go through different phases, as displayed in Figure 1. The process starts with image acquisition, which involves collecting the input images. Then, a preprocessing step is carried out to prepare the input image for the segmentation algorithm such as converting the colored images to gray-scale images, image resizing, removing noise, or color quantization. Once the image is ready, the image-segmentation process is undertaken. However, there could be some incorrect boundaries or small regions that are not segmented correctly. In order to fix these issues, image post-processing is carried out to generate the final segmented image. There are different segmentation techniques that produce different results.

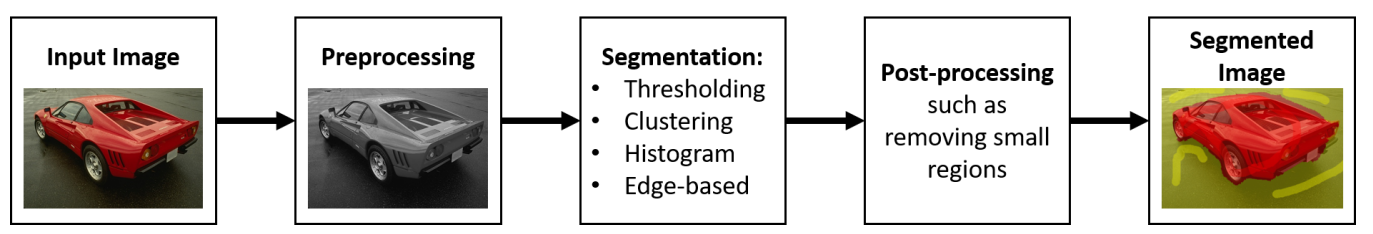

Figure 1. Image-segmentation pipeline. The car image in the pipeline is taken from the Berkeley image dataset, segmented using the Sefexa tool (http:/ / www.fexovi.com/sefexa.html, accessed on 1 January 2021).

There are several image-segmentation techniques belonging to three different types: AI techniques, segmentation techniques based on the classical method, and hybrid techniques. The main classical segmentation techniques include region-based and edge-based image segmentation. The region-based techniques include the histogram threshold, clustering, region growing, and split-and-merge methods. The AI segmentation techniques are mainly based on the ANN algorithm (for example, [14,15]), while the hybrid techniques consist of a combination of classical segmentation methods and the AI techniques (for example, [14,16]). The following section describes the four bio-inspired algorithms investigated in this study.

\section{Bio-Inspired Algorithms}

Bio-inspired algorithms are techniques developed to tackle complex problems based on biological systems of specific species [17]. Many bio-inspired algorithms are involved to enhance image segmentation, and they proved their efficiency. In this study, only four algorithms were selected: PSO [2], the GA [3], ABC [4], and ACO [5].

\subsection{Genetic Algorithm (GA)}

The GA is a bio-inspired computing method invented by [3]. It is based on the Darwinian theory of evolution that solves optimization problems by finding optimum solution or a solution adjacent to the optimum. The GA consists of a population that has specific representations (usually binary representation) composed of candidate solutions (called individuals) to an optimization problem. The algorithm consists of a set of iterations that is usually introduced by a random population of individuals. In each iteration, every individual is evaluated by means of the fitness function, and then the selection process (based on a specific selection method) is employed to select some individuals from the population. Later, crossovers and mutations are employed to reproduce a novel population for the use in the next iteration. The algorithm is based on a termination condition that might be either reaching a maximum number of iterations or a specific fitness value. Figure 2 clarifies the main steps of the codes and the algorithmic structures. For more details, the reader can refer to article [18]. 


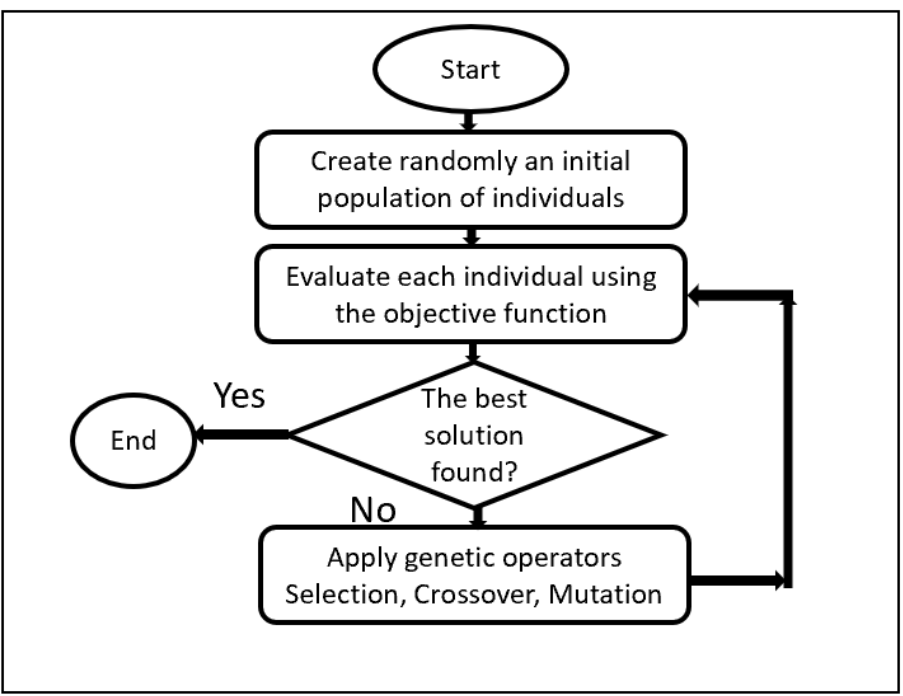

Figure 2. Flowchart of GA.

\subsection{Particle Swarm Optimization (PSO)}

PSO is a swarm intelligent technique developed by [2]. PSO is a population-based bio-inspired computing technique stimulated from the behavior of birds. It consists of a swarm (population) of particles (birds) looking for approximate solutions to optimization problems. The particles represent the solutions. The algorithm starts by randomly creating an initial swarm of particles (solutions) in the search space. The particles move into the search space based on their velocity, their current position, their best position found so far (personal best), and the position of the best particle of the swarm (global best) or the position of the best particle of the neighborhood (local best). Each particle is assessed by means of the optimization function (fitness function). Note that this article displays the classical version of PSO (without the neighborhood version).

Figure 3 simplifies the the algorithmic structures of PSO. For more details, the reader can refer to [18].

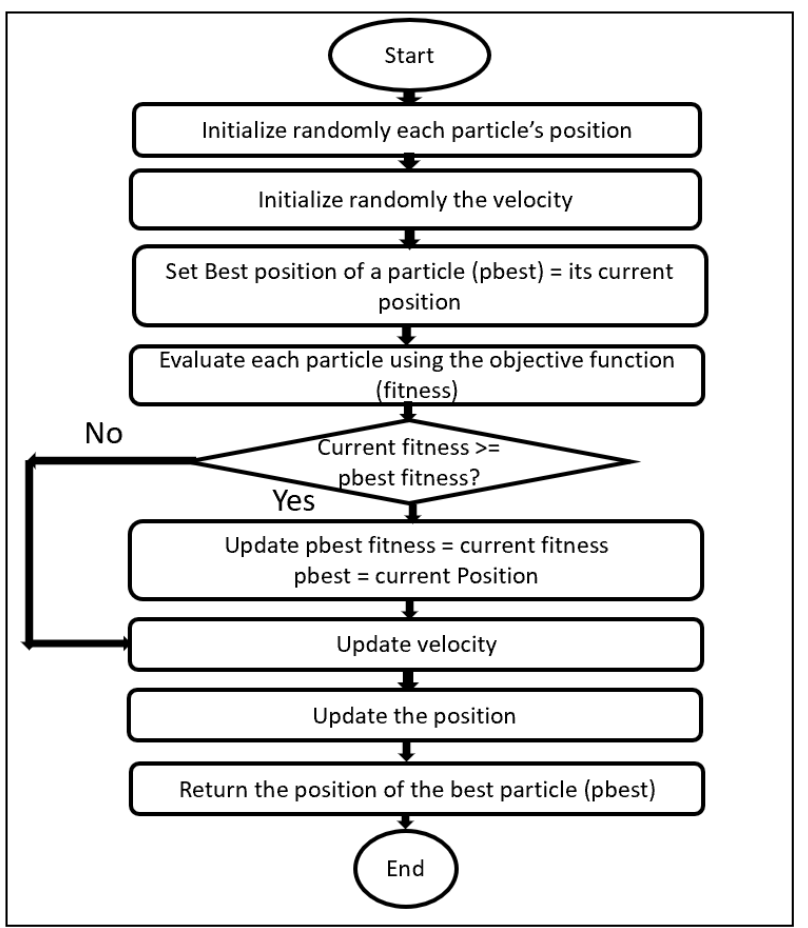

Figure 3. Flowchart of PSO. 


\subsection{Ant Colony Optimization (ACO)}

ACO was established by Marco Dorigo in 1992. It was after that improved by Dorigo et al. [5]. ACO is stimulated from real ant colonies and their way of survival. Their techniques of finding the shortest path using only their pheromones are useful to solve many problems in many areas. ACO is a population-based technique that works by having a set of artificial ants searching for an approximate solution to a specific optimization problem. To find the best solution, the studied problem is turned into a shortest-path optimization problem modeled in a weighted graph in order to use the concept of pheromones. At the beginning, each ant selects randomly a node (a vertex) and moves to another node (selected randomly) by letting pheromones behind it to form a trail. This trail attracts other ants. The more the path is taken, the more pheromone is deposited, and the more this path is attracted by the other ants. The longer path has a few quantities of pheromones because it is not frequently visited, and the few deposited pheromones are quickly evaporated. Consequently, the shortest path is quickly discovered and chosen by the ants. Figure 4 deploys the main steps of the codes. Note that, in this article, the classical version of ACO is presented.

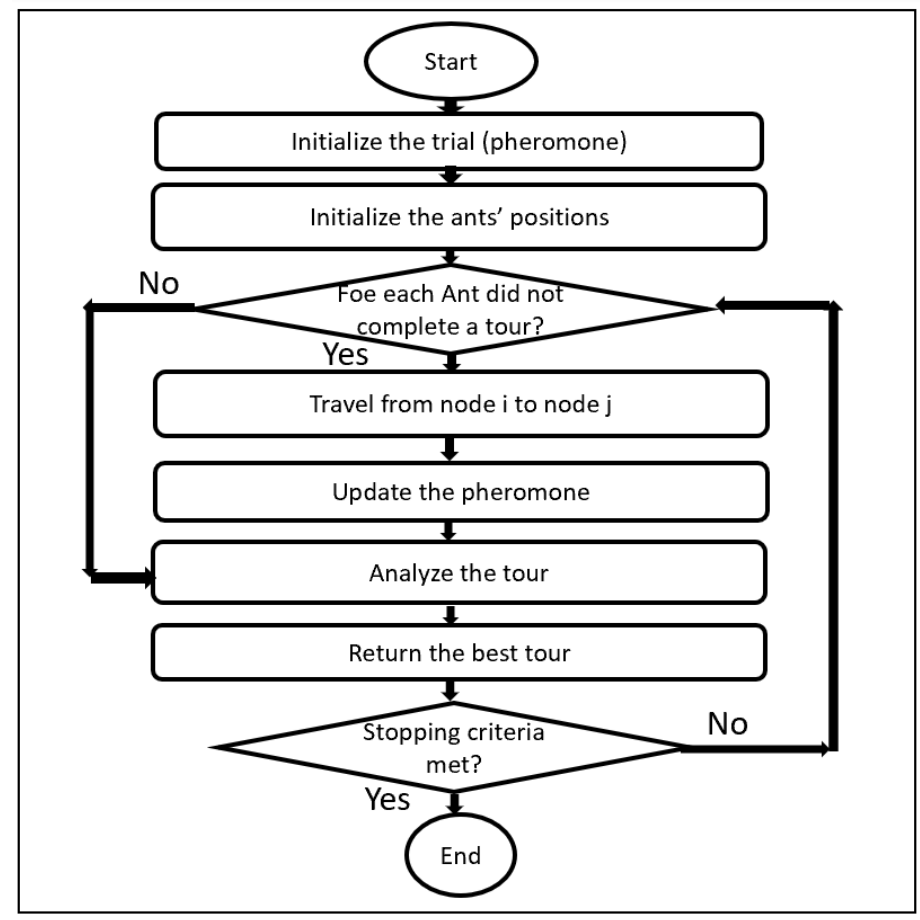

Figure 4. Flowchart of ACO.

\subsection{Artificial Bee Colony ( $A B C)$}

$\mathrm{ABC}$ was proposed in [4]. It imitates the attitude of honey-bee colonies. The bees move randomly in the search space to find food resources. In this artificial technique, the bees are categorized as onlooker bees, scout bees, and employed bees. This later type moves randomly in the search space searching for sources of nectar (food). Each employed bee will find one source of nectar (which is considered as a solution), which leads to the equality of the number of solutions and the number of employed bees. When the nectar sources are found, they are evaluated by the onlooker bees. The source of nectar having better quality (fitness value) will have a higher chance to be selected. The employed bees, which have found a high-quality food source, return to the hive to recruit scout bees by means of the waggle dance, which is considered as a method of communication between bees. Once they are attracted, the scout bees follow the employed bees to collect the best nectar sources. The solutions are the positions of the nectar sources. The swarm represents the food sources. In this algorithm, the number of scout bees is equal to the number of 
employed bees, which is exactly half of the swarm size. Then, the number of solutions is equal to half of the swarm size. The first half of the swarm represents the employed bees, while the second half consists of the onlooker bees. Figure 5 provides a simple representation of the codes.

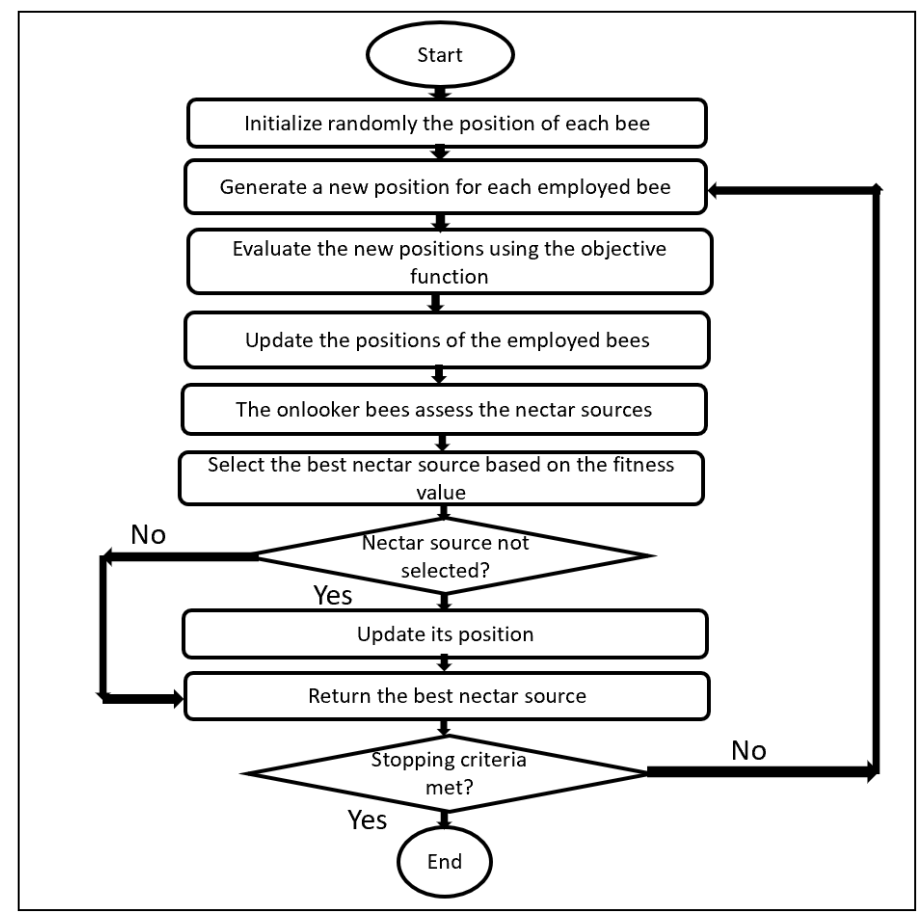

Figure 5. Flowchart of ABC.

\subsection{Adaptation of the Bio-Inspired Algorithms}

To adapt the above-mentioned techniques to solve any combinatorial problem including image segmentation, two common characteristics require definition. These characteristics depend on the problem under discussion, which are:

1. Defining the candidate solution.

2. Defining the fitness function.

Most (if not all) of the tackled optimization problems in image segmentation focus on finding the optimal threshold values in multilevel-thresholding-segmentation techniques or finding the optimal centers of the clusters in K-means or FCM clustering techniques. The following part discusses the use of these techniques in image segmentation.

\subsubsection{Thresholding Techniques}

In this problem, the candidate solution $X$ is defined as follows: $X=\left(t h r_{1}, t h r_{2}, \ldots, t h r_{q}\right)$

Where $t h r_{i}$ is the threshold $i, 0<t h r_{i}<255, i \in[0, q]$, and $q$ is the maximum number of thresholds to be set.

The fitness function is defined to maximize: the Otsu method [19], the between-class variance [19], Kapur's entropy [20], Renyi entropy [21], or Tsallis entropy [22].

\subsubsection{Clustering Techniques}

The candidate solution $X$ is defined by a $d$-dimensional vector representing the clusters' centers. $X=\left(C_{1}, C_{2}, \ldots, C_{d}\right)$ where $C_{i}$ is the center of the cluster $i, i=1, \ldots, d$, and $d$ is the number of clusters.

The fitness function is defined to minimize: the Otsu method, the between-class variance, or the fuzzy cluster validity indices [7].

The following section describes the bio-inspired-studies-related works. 


\section{Literature Review}

In the following, we present the image-segmentation studies based on a set of the bio-inspired algorithms. Firstly, the sources of the collected articles along with the search method are described. Then, the most recent studies published on bio-inspired-algorithmsbased image segmentation (such as the bat algorithm [20], the firefly algorithm [21], the elephant-herding optimization algorithm [23], and the cuckoo search [22]) are introduced. Later, a deep literature review about the four aforementioned bio-inspired-algorithmsbased segmentation (PSO, GA, ABC, and ACO) is addressed starting from 2015 until 2021.

\subsection{Sources and Search Method}

The articles were collected from 2003 to 2021. The main databases considered in this research were Elseiver, IEEE, Scopus, and Springer. The surveyed articles exceeded 110 articles, which were collected from 59 journals and 24 conferences.

Figure 6 shows the yearly distribution of the investigated articles.The number of publications in the bio-inspired image-segmentation algorithms started increasing since 2013 and achieved the highest number in 2017. To save space, only the published articles from 2015 to 2021 were discussed.

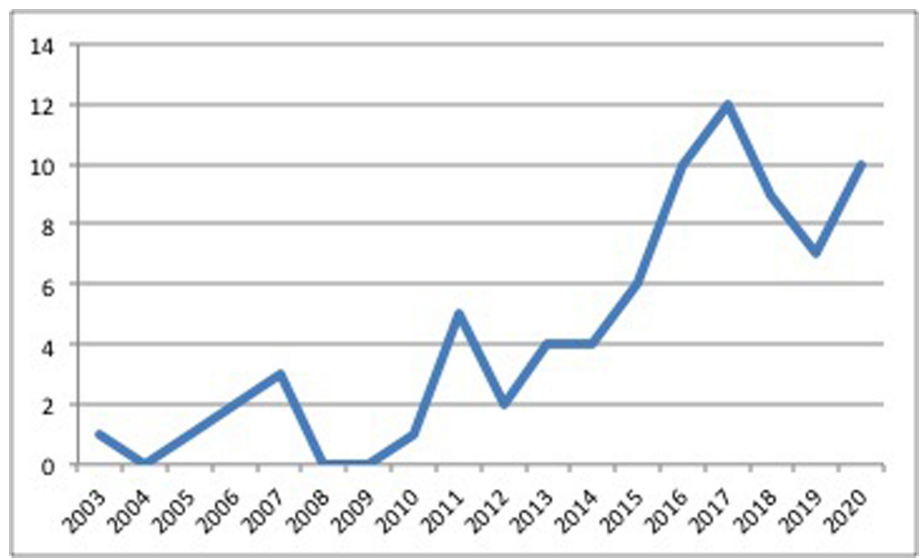

Figure 6. Yearly publication of the bio-inspired based image segmentation (2003-2021).

\subsection{Image-Segmentation-Based Bio-Inspired Algorithms}

The authors in [22] proposed a new method for segmenting the satellite images. The idea aimed at finding the optimal threshold values using the cuckoo search bio-inspired algorithm. The authors applied two objective functions including Tsallis entropy and Otsu's method. The proposed method was compared with the state-of-the art technique and validated using the peak signal-to-noise ratio (PSNR), the mean squared error (MSE), the structural-similarity-index measure (SSIM), the feature-similarity index, the universal image-quality index, and the running time. The obtained results are promising.

Besides, the authors in [20] presented a new hybrid bat algorithm to find the optimal thresholds for the multilevel-thresholding-image-segmentation method. The proposed method was combined with a genetic crossover method and inertia weight. The authors used the Otsu method with the between-class variance and the Kapur's entropy as the objective functions. The proposed method was validated using a set of images (Lena, house, bird, mountain, ruins, baboon, cottage, sailboat, couple, city, bee, and bridge) and compared with several bio-inspired algorithms. The obtained results showed that the method is competitive.

The same concept was used in [24] to find the best cluster centers in FCM. The authors proposed a combination of the modified0fuzzy-bat Algorithm (MFBA) and FCM. The fitness function was based on the between-class variance and the fuzzy-cluster-validity indices. The proposed method was validated using MRI brain images. In addition, two metrics were used including Jaccard and Dice coefficients. The comparison study showed 
that the new proposed method is competitive. Another bio-inspired method was used to enhance the image-segmentation results.

The authors in [25] used the firefly algorithm to find the best cluster centers in the Kmeans-clustering algorithm. The fitness function was represented by the Otsu's coefficient. The proposed method was tested using PET, SPECT, MRI images, and images from Harvard Whole Brain Atlas to detect the different brain tumors. Moreover, several evaluation measures were computed such as the mean error, the normalized root square, the SSIM index, and the PSNR metric. The comparison study showed that the proposed method yielded promising results.

Moreover, the authors in [23] aimed at finding the optimal threshold value at numerous levels in the multi-level thresholding method for image segmentation. They suggested the elephant-herding optimization (EHO) technique. The authors used the between-classvariance and Kapur's-entropy methods to represent the objective functions. The proposed algorithm was compared with different bio-inspired algorithms such as PSO and ABC using the Berkeley Segmentation Dataset (BSDS). Three evaluation metrics were used including the structural-similarity index (SSIM), the PSNR, and the feature-similarity index (FSIM). The results proved that EHO-based multilevel image thresholding is a competitive method.

Additionally, the authors in [21] proposed the firefly algorithm to find the optimal threshold values for image segmentation. The fitness function was represented by Renyi entropy. Three evaluation metrics were used such as the minimum, the mean, and the standard deviation. Some standard images were used to validate the proposed method. The obtained results are promising.

Furthermore in [26], the authors investigated a set of bio-inspired algorithms based on thresholding-segmentation techniques including the firefly algorithm, cuckoo search, the krill-herd algorithm, grey wolf optimization, elephant-herding optimization, the whaleoptimization algorithm, and the grasshopper-optimization algorithm. The objective function was represented by the Tsallis entropy. The authors used the medical images to perform the comparison study. The results showed that the firefly algorithm and the grey wolf optimizer outperformed the other algorithms in terms of performance in segmenting the images and convergence speed.

The same problem was discussed in [27]. The authors suggested a new bio-inspired method called dragonfly based on the thresholding-segmentation method. They used the minimum-cross-entropy and the Otsu thresholding methods. To show the performance of the proposed technique, a comparison study was performed using Berkeley datasets and some segmentation metrics (the PSNR, standard deviation, the feature-similarity index, and the SSIM index). The result is promising.

In [28], the authors employed cuckoo search and genetic algorithms to find the best parameters for edge detection. They combined interval type-2 fuzzy logic with the Sobel technique to enhance the segmentation of real-world images. The results showed that the optimized model outperformed the basic model without optimization.

\subsection{Image Segmentation Based on Genetic Algorithms (GA)}

Numerous research studies have been proposed to segment an object in an image using GA, especially to find the optimum threshold from the histogram of the image.

In [29], the authors segmented the images of moving objects (cardiac image) in image series. They used a simulated GA that applies K-means clustering in the two-dimensional feature vector space. In the experiments, a sequence of different cardiac images were registered, then only one image was utilized for the segmentation process. The results on cardiac images gave an acceptable outcome.

The authors in [30] optimized the time-consuming problem by the usage of an improved GA (IGA). They employed a fuzzy-evaluation mechanism established in the evolution process through the use of the distance to the biggest fitness value (DBFV). The 
algorithm's convergence and the entire search ability were improved compared to the basic GA. The results showed that the proposed algorithm is more effective than the basic GA.

The authors in [31] showed the effectiveness of the segmentation when applying the non-dominated sorting GA (NSGA) model using adipose-tissue images. The proposed segmentation method can come up with the best solution close to the Pareto frontier. The comparison study with the sorting GA (SGA) showed the high performance of NSGA. The authors suggested sorting the population into subsets based on the ordering of Pareto dominance to further improve the obtained result. Additionally, they suggested to compare the results with chemical-reaction optimization and $\mathrm{ACO}$ by using big data generated from a high number of equipment [32] to extract useful information from images.

A technique for exudates identification and segmentation was proposed in [33]. The exudates identification is effective in early detection of diabetic retinopathy, which is effective for saving vision and ensuring the effective treatment. The authors firstly utilized the switching median filtration method to eliminate the impact of the high-density noise produced in retinal images. Then, they applied GA to locate the exudates in retinal fundus images. The experimental results indicated that the proposed method performed better than the available techniques in terms of sensitivity, accuracy, and the error rate. The authors suggested for future work a hybridization of ACO and GA to enhance the results.

The authors in [34] suggested a combination of two methods to solve the problems of the multi-level thresholding image segmentation, including the computation time and the premature convergence. Both problems were solved by applying the GA and the gravitational-search algorithm (GSA). In GA, the roulette selection was used. The crossover and mutation rate are defined in Table 1 . The fitness function was calculated by means of the entropy and the between-class variance. The presented study was compared with the basic GSA and three GSA variants using six benchmark images. The results showed that the proposed combination was somewhat comparable to the existing works.

The authors in [35] used the GA to segment the prostate in the medical images (MRImagnetic resonance imaging) and the computed-tomography (CT) pelvic images. They proposed two- and three-dimensional automated segmentation frameworks. The GA was combined to find the shapes, the relative positions on objects, and the regional properties. The GA applied the rank selection, and the other parameters are displayed in Table 1. The fitness function was computed based on the false-alarm rate and the detection rate. To validate the results, the dice similarity metric was employed. Two experiments were presented to show the results of $2 \mathrm{D}$ and $3 \mathrm{D}$ segmentation in addition to one comparison with Chan and Vese algorithm. The obtained results were promising.

In [36], the authors proposed a hybrid-segmentation technique to detect brain cancer. They used the GA along with the discrete wavelet transform and K-means clustering. The fitness function of GA represented the clustering metric. The GA parameters are defined in Table 1. To validate the proposed method, 100 MRI brain-tumor images were used, and the accuracy and the running time were calculated. The authors did not perform a comparison study to show the effectiveness of the proposed method.

In [37], the authors presented a hybrid-segmentation method based on Tsallis and Renyi entropies in addition to the GA. The fitness function was used to maximize the entropy. The GA parameters were not mentioned. The authors used the PSNR and eight images to compare their presented method with the two-dimensional classical thresholding methods. The results were satisfactory.

The authors in [38] combined quantum GA (QGA) and differential evolution (DE) to Renyi and Tsallis entropies. The fitness function represented the maximization of the entropy. The authors did not specify the QGA parameters. The comparison studies used 22 benchmark images, and showed that DE is faster than QGA, and the proposed segmentation approaches were better than the classic thresholding image segmentation.

The authors in [39] suggested a new approach for plant leaf-disease detection. They focused on the segmentation using the GA, the K-means algorithm, and then the classification using a support vector machine (SVM). Four plant-leaf images were used to 
compare the proposed approach with the K-means algorithms only. The classification results showed that the proposed segmentation method with GA and K-means outperformed the segmentation with K-means only.

In [40], the authors proposed a combination of the GA and K-means algorithms to segment the skin-lesion regions. The fitness function involved the Jaccard index. The GA parameters were not specified. To validate the results, the skin lesions' dermoscopic images along with four metrics (the accuracy, dice, specificity, and sensitivity) were used. It was shown that the proposed method surpassed the K-means and the $\gamma$-K means.

The authors in [41] also combined the thresholding image-segmentation approach with the GA to tackle defects detection in thermographic inspection. The entropy was considered as the fitness function being maximized. Three evaluation metrics (precision, recall, and the F measure) were employed. The authors used simulated thermal images in the comparison results with five state-of-the-art studies (see Table 1). The results proved the effectiveness of the presented method.

In [42], the authors proposed a new method for apple leaf-disease identification and recognition. They used a strong correlation and expectation maximization (EM) for segmentation. To enhance the classification accuracy, they combined feature extraction with GA. The plant dataset, with thousands of images, was employed. The authors focused on enhancing the feature-extraction method instead of the segmentation.

This study presents a new fuzzy edge-detection method applied on fuzzy images. The aim of this approach was that each pixel value in a digital image can be extended to be a fuzzy number; therefore, the images can be fuzzified using interval type-2 (IT2 FS), general type-2 (GT2 FS), and type-1 fuzzy sets (T1 FS).

The authors in [43] suggested a set of meta-heuristics combined with GA and the thresholding segmentation technique. Five thresholds were tested. The authors used the roulette-wheel-selection method. To validate the results, the proposed method was compared with six swarm-intelligent methods using a set of twelve images. The authors employed four metrics including the average, the SSIM, the PSNR, and the time complexity. The obtained results were promising.

Table 1. GA-based image-segmentation techniques (published from 2003 to 2020).

\begin{tabular}{|c|c|c|c|}
\hline \multicolumn{4}{|c|}{ Population Size $(\mathrm{N})$, Crossover Probability $\left(P_{c}\right)$, Mutation Probability $\left(P_{m}\right)$, Iteration Numver (Iter) } \\
\hline Reference & Dataset & Parameter Setting & Compared Methods \\
\hline [29] & Cardiac images & Not provided & None \\
\hline [30] & MRI brain images & $\begin{array}{c}N=100 \\
P_{c}=0.8 \\
P_{m}=0.01\end{array}$ & $\begin{array}{l}\text { Exhaustive search method } \\
\text { Basic GA }\end{array}$ \\
\hline [31] & Science Photo Library & Not provided & $\begin{array}{c}\text { Sorting GA (SGA) } \\
\text { Adaptive GA (AGA) } \\
\text { Non-dominated sorting GA (NSGA) }\end{array}$ \\
\hline$[33]$ & CHASE database & Not provided & None \\
\hline [34] & Lena, Cameraman, House & Iter $=[30,3000]$ & GSA with multi-threshold \\
\hline [35] & $\begin{array}{l}\text { Berkeley Segmentation dataset } \\
\text { MRI and CT }\end{array}$ & $\begin{array}{c}N=30, P_{m}=0.1 \\
N=25 \text { and } 50 \\
P_{m}=0,1 \text { and } P_{c}=0.5\end{array}$ & $\begin{array}{l}\text { Three GSA variants with multi-level threshold } \\
\text { Chan and Vese algorithm }\end{array}$ \\
\hline [36] & MRI brain tumor & $\begin{array}{c}P_{c}=0.6, P_{m}=0.6 \\
\text { Iter }=[300,450]\end{array}$ & None \\
\hline [37] & $\begin{array}{c}\text { Grape, Peppers, Brain } \\
\text { Light microscopy and others }\end{array}$ & $\begin{aligned} \text { Iter } & =300 \\
N & =30\end{aligned}$ & 2D thresholding methods \\
\hline [38] & 22 benchmark images & Iter $=[200,400]$ & Classical thresholding method \\
\hline [39] & Plant leaf images & None & K-means \\
\hline [40] & Skin lesions demoscopic images & None & K-means and $\gamma$-K-means \\
\hline$[41]$ & Simulated thermal images & $\begin{array}{l}P_{c}=0.4, P_{m}=0.3 \\
\text { Iter }=20 \\
N=256\end{array}$ & $\begin{array}{l}\text { Otsu, Iterative Thresholding } \\
\text { K-means, Hamadani } \\
\text { Seeded Region Growing }\end{array}$ \\
\hline
\end{tabular}


Table 1. Cont.

\begin{tabular}{|c|c|c|c|}
\hline \multicolumn{4}{|c|}{ Population Size $(N)$, Crossover Probability $\left(P_{c}\right)$, Mutation Probability $\left(P_{m}\right)$, Iteration Numver $($ Iter) } \\
\hline Reference & Dataset & Parameter Setting & Compared Methods \\
\hline [42] & Plant dataset & No Applied & None \\
\hline [43] & A set of 12 images & Iter $=100, N=25$ & 6 swarm methods \\
\hline \multirow[t]{2}{*}{ [44] } & Arbitrary gray images & $N=20$ & None \\
\hline & & $\begin{array}{c}P_{c}=0.9 \\
P_{m}=0.05\end{array}$ & \\
\hline \multirow[t]{3}{*}{ [45] } & House & $N=400$ & Otsu's approach \\
\hline & Table lamp & $P_{c}=0.8$ & [19] \\
\hline & Ceiling light & $P_{m}=0.001$ & \\
\hline \multirow[t]{4}{*}{ [46] } & Rice Image & $N=10$ & None \\
\hline & Cameraman & $P_{c}=0.95$ & \\
\hline & Lifting body & $P_{m}=0.05$ & \\
\hline & Fishman & & \\
\hline [47] & Lena & $N=30$ & FHNN \\
\hline [48] & Car & $N=100$ & None \\
\hline & RGB Images & $P_{c}$ and $P_{m}$ not provided & \\
\hline [49] & MRI images & Not provided & None \\
\hline
\end{tabular}

\subsection{Image Segmentation Based on Particle Swarm Optimization (PSO)}

PSO is another bio-inspired method used in solving image segmentation.

The authors in [50] also combined a fuzzy C-mean clustering algorithm with PSO to overcome the fuzzy C-mean's shortcoming in handling the noise. PSO was used to determine the best clusters' centers values. The authors compared their proposed method to the multi-threshold Otsu image-segmentation technique and the fuzzy C-mean algorithm. However, they did not perform any quantitative comparison using some evaluation measures. In addition, only one unknown image was employed in the experiment.

The authors in [51] suggested an enhanced version of a fuzzy C-mean clustering algorithm based on PSO, spatial neighborhood information, and membership initialization. The idea aimed at applying PSO to find the best values of the clusters' centers after removing the outliers (noise) from the dataset. The proposed method was validated using brain-cancer medical images, MRI-simulated, and synthetic images. A number of evaluation measures were used including the Dice index, the Jaccard index, the truepositive fraction, the false-positive fraction, and the true-negative fraction. The proposed approach was also compared to five well-known segmentation methods: the basic fuzzy C-mean (FCM), one variant of the fuzzy C-mean (FCM S1), the robust FCM (RFCM), the kernel possibilistic C-means (KPCM), and the improved KPCM (IKPCM). The results showed that the proposed technique is a competitive segmentation method.

The authors in [52] proposed a combination of a modified version of PSO (MPSO) with two-dimensional Kullback-Leibler(K-L) divergence. The new proposed PSO involved perturbing the obtained solution using a random value in addition to the difference of the maximum and minimum particle's position. PSO was utilized to determine the best threshold with a fitness function defined as the total divergence of the regions. The distribution function included a 2D histogram. 2D K-L divergence was calculated on the original and the segmented images. To validate the results, the Berkeley segmentation and BSDS300 datasets were used. In addition, four evaluation measures were applied including the probability rand index (PRI), the variation of information (VOI), the global consistency error (GCE), and the boundary-displacement error (BDE). The authors compared their proposed method to different related methods such that 2D K-L MPSO, 1D K-L MPSO, and 1D Renyi entropy combined with MPSO and 2D K-L classic PSO. Based on the obtained results, the proposed method is effective in image segmentation.

In [52], the authors proposed a new segmentation method based on the quantum PSO (QPSO) and neutrosophic set (NS). The idea was to generate a co-occurrence matrix based 
on the transformation of the input image into the NS. The main difference of QPSO (from the classic PSO) is the absence of the velocity, in addition to the randomization involved in the calculation of the particle's position. QPSO was employed to obtain the best twodimensional threshold vector, where the fitness function is the gray-level image. The study was performed using the side scan sonar image (SSS). To validate the results, the authors compared their work to five related works using three evaluation metrics (recall, precision, and the F1 measure). The results showed an improved accuracy and execution time.

Authors in [53] proposed an image-segmentation approach based on a fast generalized FCM clustering (FGFCM) algorithm using PSO and an edge-based neighbor selection strategy. The proposed method was evaluated using the segmentation-accuracy (SA) index and the run time. Comparing the proposed method and the classic PSO declared better performance for FGFCM. However, it was time consuming (up to $4.8 \mathrm{~s}$ ) compared to FGFCM, which took 1.1 and $1.4 \mathrm{~s}$.

An enhancement of the optimal threshold selection using PSO with dynamic inertia weight (DW-PSO) was proposed in [54]. The experimental results reached the optimal threshold using DW-PSO. However, the proposed algorithm took more time than PSO as it adjusted inertia weight dynamically. The average run time was $7.3 \mathrm{~s}$ for PSO and $8.8 \mathrm{~s}$ for DW-PSO. The threshold was 98 for PSO and 102 for DW-PSO.

In [55], the rough set and the PSO algorithm were combined. The authors adopted the mean roughness measure as an evaluation standard that relied on reducing the roughness in the background regions and objects.The proposed algorithm was tested on gray-scale images (cameraman and MRI medical images).

In [56], the authors presented a new variant of PSO called chaotic Darwinian PSO (CDPSO) to enhance the image-segmentation process. The proposed method was based on deleting/adding particles depending on their performance. The fitness function involved two histogram threshold metrics (minimum cross entropy and Tsallis entropy). The proposed study was validated using satellite images (provided by Pleiades satellite imaginary) and compared with five chaotic optimization algorithms. However, no comparison was performed with existing image-segmentation methods.

The authors in [57] proposed a new variant of quantum PSO (SCQPSO) to optimize the parameters of the Otsu image-segmentation method. SCQPSO was mainly based on splitting the search space into sub-spaces allowing the inclusion of subsets of particles, to promote the best local convergence. Four stomach CT medical images were used to validate the study. The proposed method was only compared with some PSO variants showing the optimal threshold, the inter-class variance, and the standard deviation. The results showed that SCQPSO is an efficient-swarm-intelligent method. However, further investigations and comparisons could be applied to show the effectiveness of the proposed method in image segmentation.

In [58], the authors presented a new variant of PSO based on a logistic map and Sigmoid inertia weight. The idea was to promote the exploration and exploitation of the particles while preventing the premature convergence. The proposed PSO was used to improve the Otsu image-segmentation algorithm. To validate the approach, Lena and Peppers images were used. For the results, the authors showed only the original and segmented images, and no comparison was done with the image-segmentation-related works.

In [59], image segmentation with a clustering technique based on PSO was developed where a simple and clearly visualized segmentation process was done by dimension reduction for the images. The proposed technique was successfully tested on colored images and showed an increased segmentation quality.

PSO integrated with different image-segmentation features and applied for braintumor detection was discussed in [60]. The authors used brain surface extraction (BSE) to take away the skull. Then, PSO was applied to enhance the segmentation results. The local binary patterns (LBP) and deep features of the resultant image were acquired. Finally, some 
classifiers and ANN were applied after using PSO to obtain the best features selection. The experiments showed that the tumor grades can be decided with high accuracy.

In [61], the authors proposed a combination of the multi-level threshold segmentation and three PSO versions (classic PSO, Darwinian PSO (DPSO), and fractional-order PSO (FODPSO)). DPSO consisted of removing and/or creating particles based on the associated worst/best fitness value using fractional calculus. FODPSO was an extension of DPSO. The authors applied the independent component analysis before proceeding to the segmentation step. To validate the results, the Aviris Indian Pines hyper-spectral image dataset was used along with two evaluation measures including the MSE and the PSNR. The results showed that FODPSO outperformed DPSO and PSO. However, no comparison was performed with the existing works.

The authors in [62] suggested a hybrid-segmentation method based on the optical flow adaptive threshold and the PSO variant to tackle the problem of object detection and tracking in video surveillance. PSO was used to determine the best matching block in the motion estimation within the search space. This variant relied on the adaptation of the acceleration coefficients $\mathrm{C} 1$ and $\mathrm{C} 2$ to control the velocity. To validate the proposed method, three evaluation measures (Wilcoxon's rank-sum test, PSNR, and SSIM) were employed. The comparison study, using four video datasets, showed the effectiveness of this hybrid approach.

In [63], the authors applied the chaotic Darwinian PSO (CDPSO) algorithm to images compressed by the F-transform method. The main idea of using the compressed images was to decrease CPU execution time. A pre-processing stage was applied to find the ideal compression rate and the normalized comparison index. The experiments showed that the quality of the segmented images were higher than the quality gained by applying the DPSO and GA algorithms.

In [64], a three-stage method for colored images' segmentation was proposed. In the first stage, the histogram is smoothed by a Gaussian filter to remove unreliable and non-mastering peaks. In the next stage, multi-modal PSO is used to identify the peaks representing different clusters in the histogram. The algorithm can automatically determine a sufficient number of clusters. Lastly, pixels are allocated to the most-suitable cluster based on the Euclidean distance. Six quantitative assessment metrics were applied to compare the proposed method (3DHP) with FCM and SFFCM. It shows that 3DHP uses less time to find the clusters' centroids. It was also shown that all the compared methods and 3DHP have good results by means of the quantitative-evaluation function and promising segmentation results. The parameter setting values of the aforementioned studies are displayed in Table 2.

Table 2. PSO-based image-segmentation techniques (published from 2003 to 2020).

\begin{tabular}{|c|c|c|c|}
\hline \multicolumn{4}{|c|}{$\begin{array}{l}\text { Population Size }(N) \text {, Cognitive and Social, Parameters }(C 1) \text { and }(C 2) \text {, Maximum and Minimum Velocity }\left(V_{\max }, V_{\min }\right) \\
\text { Inertia }(\omega) \text {, Dimension of the Search Space }(D) \text {, Range of Gray Levels in Image (Range), Iteration Number (Iter) }\end{array}$} \\
\hline Reference & Dataset & Parameter Setting & Compared Methods \\
\hline$[50]$ & Not provided & $\begin{aligned} \text { Iter } & =200, N=100 \\
C 1 & =1.9, C 2=1.8 \\
\omega_{\max } & =0.9, \omega_{\min }=0.1\end{aligned}$ & $\begin{array}{l}\text { Fuzzy clustering } \\
\text { Multi-threshold Otsu }\end{array}$ \\
\hline [51] & $\begin{array}{c}\text { Brain Cancer Medical Images } \\
\text { Synthetic Images } \\
\text { Simulated Images }\end{array}$ & $\begin{array}{c}N=12 \\
C 1=C 2=1.70 \\
\omega=[0.4,0.9] \\
\text { Iter }=300\end{array}$ & $\begin{array}{c}\text { Fuzzy C-mean (FCM) } \\
\text { FCM-S1 } \\
\text { KPCM } \\
\text { IKPCM } \\
\text { RFCM }\end{array}$ \\
\hline [52] & $\begin{array}{l}\text { Berkeley } \\
\text { BSDS300 }\end{array}$ & $\begin{array}{c}N=20 \\
C 1=0.7, C 2=1.43 \\
\text { iter }=200\end{array}$ & $\begin{array}{c}\text { 2D D-L MPSO } \\
\text { 1D D-L MPSO } \\
\text { 2D D-L classic PSO } \\
\text { 1D Renyi entropy MPSO }\end{array}$ \\
\hline
\end{tabular}


Table 2. Cont.

Population Size (N), Cognitive and Social, Parameters (C1) and (C2), Maximum and Minimum Velocity $\left(V_{\text {max }}, V_{\text {min }}\right)$ Inertia $(\omega)$, Dimension of the Search Space $(D)$, Range of Gray Levels in Image (Range), Iteration Number (Iter)

\begin{tabular}{|c|c|c|c|}
\hline Reference & Dataset & Parameter Setting & Compared Methods \\
\hline [52] & Side Scan Sonar & $\begin{array}{l}N=20 \\
\text { Iter }=30\end{array}$ & $\begin{array}{c}\text { 2D-Otsu, MRF } \\
\text { FCM } \\
\text { Level set, fractal theory }\end{array}$ \\
\hline [53] & $\begin{array}{l}\text { Wheel image } \\
\text { Airplane } \\
\text { MRI images }\end{array}$ & $\begin{aligned} N & =50 \\
C 1 & =0.5 \\
C 2 & =0.5 \\
\text { iter } & =1000\end{aligned}$ & $\begin{array}{l}\text { Level set, fractal theory } \\
\text { Fast generalized FCM }\end{array}$ \\
\hline [54] & MRI images & $\begin{array}{c}N=20 \\
C 1=2, C 2=2 \\
V_{\text {max }}=2, V_{\text {min }}=-2 \\
\text { iter }=1000 \\
\text { Range }=[0,255]\end{array}$ & $\mathrm{PSO}$ \\
\hline [55] & $\begin{array}{l}\text { MRI images } \\
\text { Cameraman }\end{array}$ & $\begin{array}{c}N=25 \\
\text { Iter }=50\end{array}$ & Bat algorithm \\
\hline [56] & Satellite images & $\begin{array}{c}\text { Iter }=150, N=180 \\
V_{\max }=5, V_{\min }=0 \\
\omega=0,8\end{array}$ & None \\
\hline [57] & Stomach CT & $\begin{array}{c}\text { Iter }=50, N=20 \\
D=[20,100]\end{array}$ & None \\
\hline [58] & Lena \& Pepper & $\begin{array}{c}\text { Iter }=1000, D=[10,30] \\
\omega=[0.4,0.95], N=40\end{array}$ & None \\
\hline [59] & view color images & Not applied & None \\
\hline$[60]$ & $\begin{array}{c}\text { Medical images } \\
\text { from RIDER and BRATS }\end{array}$ & Not Applied & None \\
\hline [61] & $\begin{array}{c}\text { Aviris Indian Pines hyperspectral } \\
\text { image }\end{array}$ & Not provided & PSO, DPSO, FODPSO \\
\hline [62] & $\begin{array}{l}\text { Hallway } \\
\text { Foreman } \\
\text { Container } \\
\text { Coastguard }\end{array}$ & $\begin{array}{c}C 1=[0.5,2.5] \\
C 2=[0.5,2.5] \\
\text { Iter }=6\end{array}$ & $\begin{array}{c}\text { Cuckoo search } \\
\text { Flower pollination, bat } \\
\text { bird swarm } \\
\text { Gbest-guided gravitational search }\end{array}$ \\
\hline [63] & $\begin{array}{c}\text { Lena } \\
\text { Jet } \\
\text { Mandrill } \\
\text { Berkeley Segmentation Dataset } \\
\text { Image Processing Standard } \\
\text { Database }\end{array}$ & $\begin{array}{c}N=30 \\
\text { Iter }=150 \\
V_{\text {max }}=5 \\
V_{\text {min }}=0\end{array}$ & $\begin{array}{l}\text { DPSO } \\
\text { CDPSO }\end{array}$ \\
\hline [64] & $\begin{array}{l}20 \text { colour images } \\
\text { Lena }\end{array}$ & Not Applied & $\begin{array}{c}\text { FCM } \\
\text { SFFCM }\end{array}$ \\
\hline [65] & Cameraman & $\begin{array}{c}N=20 \\
C 1=1.5 \\
C 2=2 \\
V_{\max }=5 \\
V_{\min }=-5 \\
D=120 \\
\text { Range }=[0,255]\end{array}$ & $\begin{array}{c}\text { Region-based segmentation } \\
{[66]}\end{array}$ \\
\hline [67] & $\begin{array}{l}\text { House } \\
\text { Cameraman } \\
\text { Peppers }\end{array}$ & $\begin{array}{c}N=20 \\
C 1=1.49 \\
C 2=1.49 \\
V_{\text {max }}=3 \\
V_{\text {min }}=-3 \\
\text { iter }=50 \\
\text { Range }=[13,233]\end{array}$ & $\begin{array}{l}\text { Normal region-based } \\
\text { segmentation } \\
\text { K-means algorithm }\end{array}$ \\
\hline [68] & MIAS database $\left(^{*}\right)$ & Not provided & $\begin{array}{l}\text { PSO, Darwinian PSO (DPSO) } \\
\text { Fractional-order DPSO (FO-DPSO) }\end{array}$ \\
\hline [69] & Arbitrary RGB Images & Not provided & None \\
\hline
\end{tabular}




\subsection{Image Segmentation Based on Ant Colony Optimization (ACO)}

Numerous research studies have been proposed to segment an object in an image using ACO.

In [70], the authors proposed ACO-based edge detection to overcome the limitations of the conventional methods applied to recognize the number of plates. The suggested technique was efficient in creating a pheromone table (matrix) that presents the edge information shown at each image's pixel position, which is determined based on the ants' movements on the image. Additionally, ants' movements were conducted by the local fluctuation of the image's intensity values. The results showed that $92.6 \%$ of the number of plate characters were successfully recognized. However, the recognition errors appeared in similar-looking characters.

Using the ACO technique is usually time consuming. The authors in [71] proposed to improve the execution time by combining the multi-threading with ACO for solving edge-detection problems. The proposed method aimed at increasing the randomness among the used artificial ants by implementing the ants as threads that run in parallel. This work resulted in a new method with a smaller number of steps that reduced the time.

In [72], the authors proposed to use GA to find the best initial solution for ACO in the thresholding problem. The proposed method improved the resulted thresholds for the 2D-fisher-criteria-based thresholding method. Even though it was slower than the traditional GA, it yielded better fitness. It also outperformed the 2D Otsu method with images containing a single object.

In [73], the authors utilized ACO in order to detect the cup region in retinal images for glaucoma detection. The image segmentation was done by combining the intensity gradient of the optic disc area and the curvature of the vessels. The proposed method was successfully compared against other techniques. The results yielded an average overlapping error of $24.3 \%$.

The authors in [74] proposed a new MR-image-segmentation technique using an ACO algorithm to identify the cluster centers of the FCM clustering and to determine the number of its clusters. The results improved the speed of clustering and avoided falling into local minimums. In addition, it was shown that the quality of segmentation and the anti-noise capabilities were enhanced.

The authors in [75] proposed a modified ACO to detect malignant cells of prostate biopsies and compared them with manual detection methods. The results showed a high performance of the proposed method against the manual methods with an improvement rate ranging between 0.3 and 0.56 .

In [76], the ACO algorithm was used for feature selection in the proposed hybrid aerial image-segmentation algorithm. The ACO identified the 12 most-important features out of 31 features, which were used to improve the extraction of date palm trees from images. The use of ACO for feature selection improved the overall performance accuracy (to reach $88 \%$ ) compared to other feature-selection algorithms.

The authors in [77] used an ACO technique to improve the quality of edge detection in the ship images under the sea water. It was combined with the triangular fuzzy membership function to decide which pixels represent edge pixels in the images. The proposed method performed better than the conventional FCM-based ACO algorithm.

The authors in [78] proposed to use ACO to determine the contours of objects on complex structured color images through the movement of ants. The proposed method was compared against other methods using the first and second kind of errors. The results showed that the proposed ACO method in determining the contours of objects achieved an error rate ranging between $5 \%$ and $13 \%$.

In [79], the authors proposed a hybrid optimized neural classifier (HONC) to classify the retinal images and to detect blood vessels. The proposed model used ANN backtracking propagation. Then, it was improved using a set of optimized features, in the classification stage, which were extracted using ACO as a pre-processing step. The proposed method 
enhanced the accuracy by $14 \%$ compared to FCM, $11.01 \%$ compared to BSM, and $4.32 \%$ compared to ANN methods.

In [80], the authors proposed to use ACO and textural features to optimize the MRI brain-image segmentation using the thresholding technique. The proposed method expanded the range of ant exploration, which enabled a better segmentation of the brain tissues. The authors used many MRI images and six evaluation measures (the false-positive rate (FPR), the false-negative rate (FNR), the mean accuracy, the false-discovery rate (FDR), the false-omission rate (FOR), and precision metrics) to test and compare the proposed technique. The experimental study showed promising results.

The parameter setting values of the aforementioned studies are displayed in Table 3.

Table 3. ACO based image segmentation techniques (Published from 2003 to 2020).

\begin{tabular}{|c|c|c|c|}
\hline \multicolumn{4}{|c|}{$\begin{array}{l}\text { Number of Iterations }(\text { Iter), Number of Ants }(N) \\
\text { Evaporation of the Pheromone Trail }(\rho) \text {, Initial Pheromone Quantity }\left(\tau_{0}\right) \\
\text { Maximum Pheromone Quantity }\left(\tau_{\max }\right) \text {, the Relative Importance of the Residual Pheromones }(\alpha) \\
\text { the Relative Importance of the Pheromone Trails }(\beta) \text {, the Pheromone Decay Coefficient }(\phi)\end{array}$} \\
\hline Reference & Dataset & Parameter Setting & Compared Methods \\
\hline [70] & Vehicle image & Not provided & $\begin{array}{l}\text { SOBEL operator [81] } \\
\text { Prewitt operator [82] } \\
\text { ResultACOoberts's } \\
\quad \text { operator [83] }\end{array}$ \\
\hline$[71]$ & $\begin{array}{l}\text { Shapes } \\
\text { Lena } \\
\text { Mandril } \\
\text { Cameraman } \\
\text { Chairs } \\
\text { Pepper }\end{array}$ & $\begin{array}{c}\text { iter }=3 \\
N=\sqrt{w * h} \\
\text { (i.e., } w * h \text { is the number of pixels) } \\
\tau_{0}=0.0001 \\
\alpha=1 \\
\beta=0.1\end{array}$ & Conventional ACO \\
\hline$[72]$ & $\begin{array}{l}\text { Person } \\
\text { Rice } \\
\text { Man } \\
\text { Tank }\end{array}$ & $\begin{array}{c}\text { iter }=5 \\
N=5 \\
\alpha=1.0 \\
\beta=1.0 \\
\rho=[0,1]\end{array}$ & $\begin{array}{c}\text { 2D Otsu } \\
\text { Traditional GA }\end{array}$ \\
\hline [73] & RIM-ONE dataset & $\begin{aligned} \text { iter } & =250 \\
N & =50 \\
\alpha & =3 \\
\beta & =3\end{aligned}$ & $\begin{array}{c}\text { Thresholding } \\
\text { R-bend } \\
\text { ASM } \\
\text { Regression } \\
\text { Superpixel }\end{array}$ \\
\hline [74] & Medical MRI images & Not provided & $\begin{array}{c}\text { FCM } \\
\text { Markov random field (MRF) }\end{array}$ \\
\hline [75] & MRI-guided prostate biopsies & Not provided & Manual method \\
\hline [76] & $\begin{array}{l}\text { Very-high-spatial-resolution } \\
\text { (VHSR) aerial imagery } \\
\text { provided by the } \\
\text { Ajman municipality }\end{array}$ & Not provided & $\begin{array}{c}\text { Chi-square } \\
\text { CFS } \\
\text { Gain ratio } \\
\text { Information gain } \\
\text { SVM } \\
\text { Principal component } \\
\text { analysis (PCA) }\end{array}$ \\
\hline [77] & $\begin{array}{l}\text { Infrared image of } \\
\text { ship }\end{array}$ & $\begin{array}{l}N=4 \\
\tau_{0}=0.001 \\
\rho=0.01 \\
\phi=0.001\end{array}$ & FCM baed ACO \\
\hline [78] & Structured color image & $\begin{array}{c}\text { iter }=50 \\
N=735196 \\
\begin{array}{c}\alpha=2 \\
\beta=1 \\
\rho=10^{-3} \\
\tau_{0}=10^{-3}\end{array}\end{array}$ & $\begin{array}{l}\text { Canny method } \\
\text { K-means method }(k=2, k=3) \\
\text { Random forest method }\end{array}$ \\
\hline
\end{tabular}


Table 3. Cont.

\begin{tabular}{|c|c|c|c|}
\hline \multicolumn{4}{|c|}{$\begin{array}{l}\text { Number of Iterations }(\text { Iter }), \text { Number of Ants }(N) \\
\text { Evaporation of the Pheromone Trail }(\rho) \text {, Initial Pheromone Quantity }\left(\tau_{0}\right) \\
\text { Maximum Pheromone Quantity }\left(\tau_{\max }\right) \text {, the Relative Importance of the Residual Pheromones }(\alpha) \\
\text { The Relative Importance of the Pheromone Trails }(\beta) \text {, the Pheromone Decay Coefficient }(\phi)\end{array}$} \\
\hline Reference & Dataset & Parameter Setting & Compared Methods \\
\hline [79] & $\begin{array}{l}\text { DRIVE database } \\
\text { STARE database }\end{array}$ & Not provided & $\begin{array}{c}\text { Bayesian set models } \\
\text { Standalone ANN models }\end{array}$ \\
\hline$[80]$ & BrainWeb database & Not provided & $\begin{array}{c}\text { FCM } \\
\text { Expectation-maximization (EM) } \\
\text { Improved bacterial foraging } \\
\text { Algorithm (IBFA) } \\
\text { Improved PSO } \\
\text { Optimization (IPSO) }\end{array}$ \\
\hline$[84]$ & $\begin{array}{l}\text { Cerebral MRI } \\
\text { Resonance (MR) }\left(^{*}\right) \\
\text { House }\end{array}$ & $\begin{array}{l}\text { iter }=2500 \\
N=10 \\
\rho=0.9 \\
\tau_{0}=0.001\end{array}$ & $\begin{array}{c}\text { Simulated annealing (SA) } \\
\text { GA }\end{array}$ \\
\hline [85] & $\begin{array}{c}\text { Cerebral MRI } \\
\text { Resonance (MR) }\left(^{*}\right) \\
\text { Muscle cells image } \\
\text { House }\end{array}$ & $\begin{array}{l}\text { iter }=2500 \\
\quad N=10 \\
\rho=0.01 \\
\alpha=0.01 \\
\beta=2\end{array}$ & $\begin{array}{l}\text { Simulated annealing (SA) } \\
\text { GA } \\
\text { ACS-MRF }\end{array}$ \\
\hline [86] & $\begin{array}{c}\text { Banana } \\
\text { RGB images }\end{array}$ & $\begin{array}{l}\rho=0.5 \\
\alpha=1 \\
\beta=5\end{array}$ & None \\
\hline [87] & $\begin{array}{l}\text { Two-dimensional } \\
\text { electrophoresis gel images }\end{array}$ & $\begin{array}{c}\rho=0.01 \\
\tau_{0}=0.07 \\
\alpha=0.025 \\
\beta=3.5\end{array}$ & None \\
\hline [88] & RGB images & $\begin{array}{l}\alpha=1 \\
\beta=5\end{array}$ & $\begin{array}{c}\text { Sobel approach } \\
\text { Canny approach } \\
\text { Watershed approaches }\end{array}$ \\
\hline [89] & Heart ventricle image & $\begin{array}{c}\text { iter }=48 \\
N=10 \\
\tau_{\operatorname{Max}}=50 \\
\alpha=5 \\
\beta=0.1\end{array}$ & $\begin{array}{c}\text { Original ACO } \\
\text { GA }\end{array}$ \\
\hline [90] & Pepper & $\begin{array}{l}\text { iter }=30 \\
\rho=0.5 \\
\tau_{0}=1 \\
\alpha=2 \\
\beta=1\end{array}$ & Original ACO \\
\hline
\end{tabular}

(*) http:/ / www.bic.mni.mcgill.ca/brainweb, accessed on 10 March 2019.

\subsection{Image Segmentation Based on Artificial Bee Colony ( $A B C)$}

$\mathrm{ABC}$ is another bio-inspired algorithm greatly employed in image segmentation.

In order to speed up the multilevel-thresholding-based image segmentation, ABC was used as a pre-processing step in [91]. The aim was to determine the best threshold distribution and improve the quality of detecting the iris in images. The authors tested the approach with and without this pre-processing step. It was noted that the detection rate became more accurate after using the $\mathrm{ABC}$ as a pre-segmentation step.

In the work of [92], image segmentation was handled as a multiobjective optimization problem by combining an improved $\mathrm{ABC}$ algorithm with seeded region growth. The segmentation was considered as a clustering problem. The process started from seeds determined by optimizing a set of cluster validity indices. Then, it terminated when similar small and large clusters are grouped together. The results showed that the performance of the proposed method had a high accuracy, and it was consistent over multiple runs. 
The proposed method in [93] combined the ABC algorithm with FCM to define the FABC segmentation algorithm. The proposed method used ABC to search for the optimal cluster centers by utilizing fuzzy membership functions. The authors compared the proposed FABC with PSO, the GA, and EM. They concluded that it outperformed the existing studies, and it was more stable in terms of convergence, computational cost, speed, and accuracy.

In [94], the authors modified the objective function to use the Kapur's entropy in the modified quick ABC algorithm (MQABC) to perform the multilevel thresholding in color-image segmentation. The difference of the proposed method and the standard ABC algorithm focused on the search for new food sources performed by the employed and onlooker bees. The proposed MQABC method was compared against electro-magnetism optimization (EMO), classical ABC, and modified discrete grey wolf optimizer (MDGWO). MQABC achieved the optimal thresholds faster than its counterparts with a good segmentation quality.

The authors of [95] proposed an improved ABC method that utilized quantum and space coordinates to find the optimal segmentation threshold of the images. The proposed method (BQABC) used Bloch spherical coordinates to encode the cubits in order to initialize the food sources. The BQABC method was compared against other methods. The BQABC algorithm has proved to converge all the time, while the other methods failed to converge at least two to five times. It had better and stable performance.

In [96], the authors enhanced the accuracy of detecting the white blood cells by applying $\mathrm{ABC}$. The proposed technique was based on two steps. The first step was image segmentation using an ABC-based Otsu-threshold-segmentation method. The second step was ellipse detection to find the best ellipse candidate. The proposed approach was compared with the differential evolution (DE) and PSO. The results obtained by PSO and $\mathrm{ABC}$ were similar but better than DE. However, in the multi-dimensional problems, ABC outperformed DE and PSO. The proposed method was more accurate and efficient.

The authors in [97] applied the FCM combined with four-chain quantum bee colony optimization (QABC). This later contributed in the initialization of the clustering centers and reduced the noisy data. The experiments on the proposed method (FQABC) were performed 10 times to remove the impact of factors randomization, and the resulted values were averaged. The results showed that the computational time of FQABC was reduced, and the convergence was obtained after 30 iterations. Unlike FQABC, FABC (FCM combined with $\mathrm{ABC}$ ) required more than 30 iterations. The reason was that the FQABC algorithm used the enhanced QABC algorithm that determined the best initial FCM clustering centers. FQABC highly reduced the running time of FCM and decreased the computation complexity.

Another modified ABC technique was proposed to perform the multilevel thresholding for color image segmentation [98]. It used the krill herd algorithm in the onlooker-bees phase to find the thresholds of the feature vector (called KABC). Moreover, PSO was used to initialize the population with the best solution. The resulting method (called ABCKP) improved the optimization accuracy and the convergence speed. The authors compared the performance metrics (the boundary displacement error (BDE), the PSNR, and the featuresimilarity-index measurement (FSIM)) to find the similarity of region-contours-segmented images and the ground truth. They found that ABCKP achieved an improvement rate of $16.00 \%, 6.47 \%$, and $7.05 \%$ with the respective measures.

The ABC algorithm was also used by [99] to improve the pattern recognition and the clustering quality of the proposed kernel FCM (KFCM) method. The ABC algorithm was utilized to improve the learning patterns in images by optimizing the pattern-membership scaling function. The KFCM method performed well compared to the K-means, FCM, and spatial FCM methods. It demonstrated an improvement in the accuracy up to $0.8-5.5 \%$ compared to other counterparts methods.

The authors in [100] utilized the ABC algorithm to improve the edge-detection quality in images. They employed the $\mathrm{ABC}$ algorithm for finding the optimal coefficients for the 
edge filter and for optimizing the threshold value in image segmentation. The results showed that the $A B C$ algorithm was effective in producing high-quality solutions for edge detection compared to the state-of-the-art techniques.

In [101], the authors proposed a new hybrid $A B C$ (HABC) technique to perform the multilevel image segmentation. The optimal thresholds were obtained after combining the $\mathrm{ABC}$ algorithm with the life-cycle foraging rules, the orthogonal Latin squares approach, and the crossover. The $\mathrm{ABC}$ and the proposed $\mathrm{HABC}$ algorithms were tested and compared with other methods such as an adaptive bacterial foraging optimizer with life-cycle and social learning (BFLOS), a covariance-matrix-adaptation-evolution strategy (CMA_ES), and PSO. Both techniques ( $\mathrm{ABC}$ and $\mathrm{HABC}$ ) performed better than their other counterpart algorithms in most tests. The HABC method was more efficient than the $\mathrm{ABC}$ algorithm because of its optimal searching strategies.

In [102], the authors proposed to improve the search performance in a novel proposed method called SCABC. The process was based on replacing the employed bees phase of the classical $\mathrm{ABC}$ algorithm with the modified search equations of the sine-cosine algorithm (SCA). The proposed method was compared against other methods using 23 well-known benchmark datasets. They performed statistical, convergence, and performance-index analysis. The proposed hybrid SCABC outperformed the other methods, especially in the uni-modal test problems. It also demonstrated good performance in multi-modal problems. Moreover, the proposed hybrid SCABC was successfully compared against the standard $\mathrm{ABC}$ and SCA to perform multilevel gray-image thresholding using Kapur's entropy as the objective function.

The authors in [103] modified the updating strategy of the ABC algorithm by using the block spherical coordinates of a cubit combined with Kapur's entropy. The aim was to find the optimal thresholds in the gray-image multilevel-threshold segmentation. The proposed algorithm was superior compared to the existing algorithms in terms of convergence, speed, and overall performance.

In [104], the authors compared ABC-based segmentation against the K-means, FCM, and GA methods to extract the boundary of brain tumors in MRI. They concluded that segmenting the images using evolutionary methods outperformed the traditional clustering methods such as K-means and FCM. When comparing the evolutionary methods, the ABC algorithm yielded better performance than the GA in all the segmentation cases.

In [105], the authors proposed a modified version of the $A B C$ algorithm to handle image segmentation. The idea consisted of modifying the search behavior of $\mathrm{ABC}$ by including the mean of the previous best solutions to the search equation. This proposed MeanABC was successfully compared against many variants of $A B C$ techniques in teams of running time, performance, and segmentation quality. This Mean $A B C$ was also combined with the FCM algorithm to segment a set of 20 volumes of real brain MRI scans with 20 images in each volume. The hybrid approach was compared with other state-of-the-art segmentation algorithms and has shown to be effective in finding the optimum solution.

In [106], the authors combined FCM with the ABC algorithm to segment gray images. In order to speed up the calculation of the optimal clusters in the segmented image, fuzzy membership functions were used. The authors also utilized negative logarithm values to avoid the sensitivity to noise and outliers in the images. The proposed algorithm has shown to be effective in extracting the main objects as well as the detailed information of the image. However, it has some performance limitations in the presence of complex backgrounds.

The parameter setting values of the aforementioned studies are displayed in Table 4 . 
Table 4. ABC-based image-segmentation techniques (published from 2003 to 2020).

\begin{tabular}{|c|c|c|c|}
\hline \multicolumn{4}{|c|}{$\begin{array}{c}\text { Number of Iterations (Iter), Number of Ants }(N) \text {, Evaporation of the Pheromone Trail }(\rho) \text {, Initial Pheromone Quantity }\left(\tau_{0}\right) \\
\text { Maximum Pheromone Quantity }\left(\tau_{\max }\right) \text {, the Relative Importance of the Residual Pheromones }(\alpha) \\
\text { the Relative Importance of the Pheromone Trails }(\beta) \text {, the Pheromone Decay Coefficient }(\phi)\end{array}$} \\
\hline Reference & Dataset & Parameter Setting & Compared Methods \\
\hline$[70]$ & Vehicle image & Not provided & $\begin{array}{l}\text { SOBEL operator [81] } \\
\text { Prewitt operator [82] } \\
\text { ResultACOoberts's } \\
\text { operator [83] }\end{array}$ \\
\hline [71] & $\begin{array}{l}\text { Shapes } \\
\text { Lena } \\
\text { Mandril } \\
\text { Cameraman } \\
\text { Chairs } \\
\text { Pepper }\end{array}$ & $\begin{array}{c}\text { iter }=3 \\
N=\sqrt{w * h} \\
\text { (i.e., } w * h \text { is the number of pixels) } \\
\tau_{0}=0.0001 \\
\alpha=1 \\
\beta=0.1\end{array}$ & Conventional ACO \\
\hline [72] & $\begin{array}{l}\text { Person } \\
\text { Rice } \\
\text { Man } \\
\text { Tank }\end{array}$ & $\begin{array}{c}\text { iter }=5 \\
N=5 \\
\alpha=1.0 \\
\beta=1.0 \\
\rho=[0,1]\end{array}$ & $\begin{array}{c}\text { 2D Otsu } \\
\text { Traditional GA }\end{array}$ \\
\hline$[73]$ & RIM-ONE dataset & $\begin{aligned} \text { iter } & =250 \\
N & =50 \\
\alpha & =3 \\
\beta & =3\end{aligned}$ & $\begin{array}{l}\text { Thresholding } \\
\text { R-bend } \\
\text { ASM } \\
\text { Regression } \\
\text { Superpixel }\end{array}$ \\
\hline [74] & Medical MRI images & Not provided & $\begin{array}{l}\text { FCM } \\
\text { MRF }\end{array}$ \\
\hline [75] & MRI-guided prostate biopsies & Not provided & Manual method \\
\hline [76] & $\begin{array}{l}\text { Very-high-spatial-resolution } \\
\text { (VHSR) aerial imagery } \\
\text { provided by the } \\
\text { Ajman municipality }\end{array}$ & Not provided & $\begin{array}{c}\text { Chi-square } \\
\text { CFS } \\
\text { Gain ratio } \\
\text { Information gain } \\
\text { SVM } \\
\text { Principal component } \\
\text { analysis (PCA) }\end{array}$ \\
\hline [77] & $\begin{array}{l}\text { Infrared image of } \\
\text { ship }\end{array}$ & $\begin{aligned} N & =4 \\
\tau_{0} & =0.001 \\
\rho & =0.01 \\
\phi & =0.001\end{aligned}$ & FCM based ACO \\
\hline [78] & Structured color image & $\begin{array}{c}\text { iter }=50 \\
N=735196 \\
\begin{array}{c}\alpha=2 \\
\beta=1 \\
\rho=10^{-3} \\
\tau_{0}=10^{-3}\end{array}\end{array}$ & $\begin{array}{c}\text { Canny method } \\
\text { K-means method }(k=2, k=3) \\
\text { Random forest method }\end{array}$ \\
\hline [79] & $\begin{array}{l}\text { DRIVE database } \\
\text { STARE database }\end{array}$ & Not provided & $\begin{array}{c}\text { Bayesian set models } \\
\text { Standalone ANN models }\end{array}$ \\
\hline [80] & BrainWeb database & Not provided & $\begin{array}{c}\text { FCM } \\
\text { Expectation-maximization (EM) } \\
\text { Improved bacterial foraging } \\
\text { algorithm (IBFA) } \\
\text { Improved particle swarm } \\
\text { optimization (IPSO) }\end{array}$ \\
\hline [84] & $\begin{array}{l}\text { Cerebral MRI } \\
\text { resonance }(\mathrm{MR})\left(^{*}\right) \\
\text { House }\end{array}$ & $\begin{array}{l}\text { iter }=2500 \\
\qquad \begin{array}{c}N=10 \\
\rho=0.9 \\
\tau_{0}=0.001\end{array}\end{array}$ & $\begin{array}{c}\text { Simulated annealing (SA) } \\
\text { GA }\end{array}$ \\
\hline
\end{tabular}


Table 4. Cont.

\begin{tabular}{|c|c|c|c|}
\hline \multicolumn{4}{|c|}{$\begin{array}{c}\text { Number of Iterations (Iter), Number of Ants }(N) \text {, Evaporation of the Pheromone Trail }(\rho) \text {, Initial Pheromone Quantity }\left(\tau_{0}\right) \\
\text { Maximum Pheromone Quantity }\left(\tau_{\max }\right) \text {, the Relative Importance of the Residual Pheromones }(\alpha) \\
\text { The Relative Importance of the Pheromone Trails }(\beta) \text {, the Pheromone Decay Coefficient }(\phi)\end{array}$} \\
\hline Reference & Dataset & Parameter Setting & Compared Methods \\
\hline \multirow[t]{4}{*}{ [85] } & Cerebral MRI & iter $=2500$ & Simulated annealing (SA) \\
\hline & Resonance (MR) ${ }^{*}$ ) & $N=10$ & GA \\
\hline & Muscle cells image & $\rho=0.01$ & ACS-MRF \\
\hline & House & $\begin{array}{c}\alpha=0.01 \\
\beta=2\end{array}$ & \\
\hline \multirow[t]{2}{*}{ [86] } & Banana & $\rho=0.5$ & None \\
\hline & RGB images & $\begin{array}{l}\alpha=1 \\
\beta=5\end{array}$ & \\
\hline \multirow[t]{2}{*}{ [87] } & Two-dimensional & $\rho=0.01$ & None \\
\hline & electrophoresis gel images & $\begin{array}{l}\tau_{0}=0.07 \\
\alpha=0.025 \\
\beta=3.5\end{array}$ & \\
\hline \multirow[t]{2}{*}{ [88] } & RGB images & $\alpha=1$ & Sobel approach \\
\hline & & $\beta=5$ & $\begin{array}{c}\text { Canny approach } \\
\text { Watershed approaches }\end{array}$ \\
\hline \multirow[t]{3}{*}{ [89] } & Heart ventricle image & iter $=48$ & Original ACO \\
\hline & & $N=10$ & GA \\
\hline & & $\begin{array}{l}\tau_{\operatorname{Max}}=50 \\
\quad \alpha=5 \\
\beta=0.1\end{array}$ & \\
\hline$[90]$ & Pepper & iter $=30$ & Original ACO \\
\hline & & $\rho=0.5$ & \\
\hline & & $\tau_{0}=1$ & \\
\hline & & $\alpha=2$ & \\
\hline & & $\beta=1$ & \\
\hline
\end{tabular}

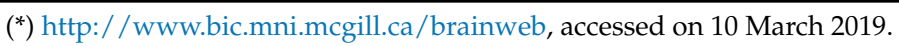

\section{Discussion}

This section is the heart of this article. It discusses the main concept of bio-inspired algorithm-based image segmentation. Firstly, the main segmentation techniques introduced in this review were highlighted. Then, the mostly used bio-inspired algorithms were emphasized. Later, a long discussion about the parameter setting of the AI methods is provided. This part might be helpful for any non-expert in AI. Next, the well-known datasets and the common evaluation metrics used in image segmentation are exhibited. Additionally, some tools and programming languages utilized in image segmentation are introduced. Finally, pertinent suggestions and new perspectives are recommended.

\subsection{Segmentation Techniques}

The four AI methods were mainly combined with the thresholding techniques, clustering techniques, edge-detection techniques, and histogram segmentation techniques. The most central part for implementing any of these segmentation approaches is to determine the appropriate threshold value and the number of clusters. These parameters have to be set by the researcher taking into account the implemented algorithm and the problem tackled. The AI methods were called to solve this matter. For the thresholding and histogram segmentation methods, the AI methods were hybridized with the entropy. According to Pare et al. [107], the most-popular and commonly used histogram-based entropy functions are Kapur, Otsu, and Tsalli entropies for both the gray-scale and colored images. The aim was to find out the best value of the threshold levels. The AI methods were utilized to find the peaks and valleys of the image histogram in order to determine the optimal threshold values, which are used in the image-segmentation problem. 
In the clustering segmentation techniques, the four techniques were implemented with FCM and K-means clustering techniques in order to find out the optimal number of clusters and their centers. The edge-detection segmentation technique was only combined with ACO to determine the edge information based on the ants' movements. Most of the related studies successfully compared their proposed combined approaches with the basic image-segmentation techniques.

\subsection{Bioinspired Techniques}

The bio-inspired algorithms were successfully combined with the segmentation techniques to enhance the quality of the segmented images. Figure 7 displays the number of times these methods were applied in solving image segmentation in these recent seventeen years. As noticed, the ABC algorithm exceeded expectations. The GA and PSO are mutually used, and ACO was less frequently applied.

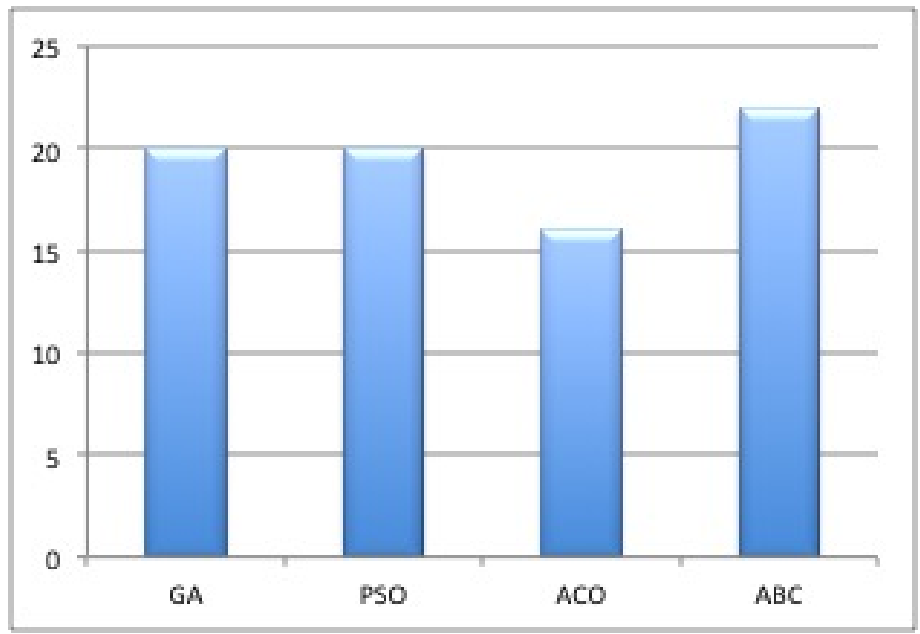

Figure 7. Number of publications for each bio-inspired method (2003-2020).

Figure 8 shows the yearly publication frequency of the four bio-inspired algorithmsbased image segmentation. As seen, the first use of an ABC method is recent (in 2011). Then, its use increased since 2017. Most of the proposed ABC methods aimed at handling the multilevel threshold image segmentation. The approach yielded encouraging results. However, some recommendations have been mentioned such as optimizing the initial generation of the $\mathrm{ABC}$ population and improving the speed.

The GA is another bio-inspired technique, which has been employed since 2006 in image segmentation. Then, its utilization has been augmented since 2015. GAs are combined with the thresholding, clustering, filtering, and histogram approaches to find the optimal thresholds and the best cluster centers. As noticed, GAs have not been extremely used in image segmentation compared to $A B C$ in these recent seventeen years (see Figure 7) despite the promising obtained results. GAs are well-known bio-inspired methods used in many domains to solve hard and complex optimization problems. So, it is better to improve this approach and exceed its use in this domain.

PSO is an evolving digital image-segmentation technique. It was applied in these last seventeen years starting from 2010 and has been increasingly employed since 2015, as shown in Figure 8. PSO was combined with different clustering methods to find regions into the images and has obtained promising results. Several authors recommended using PSO in medical-image segmentation and combining it with the ANN technique.

ACO has mostly not been applied. It has been used since 2003 but with low frequency. ACO was combined with the clustering techniques (FCM and K-means) to determine the optimal cluster centers. It was also combined with edge detection to define the edges through the ants' movement. The obtained results were efficient in terms of quality, but it is time consuming due to the pheromone update. 


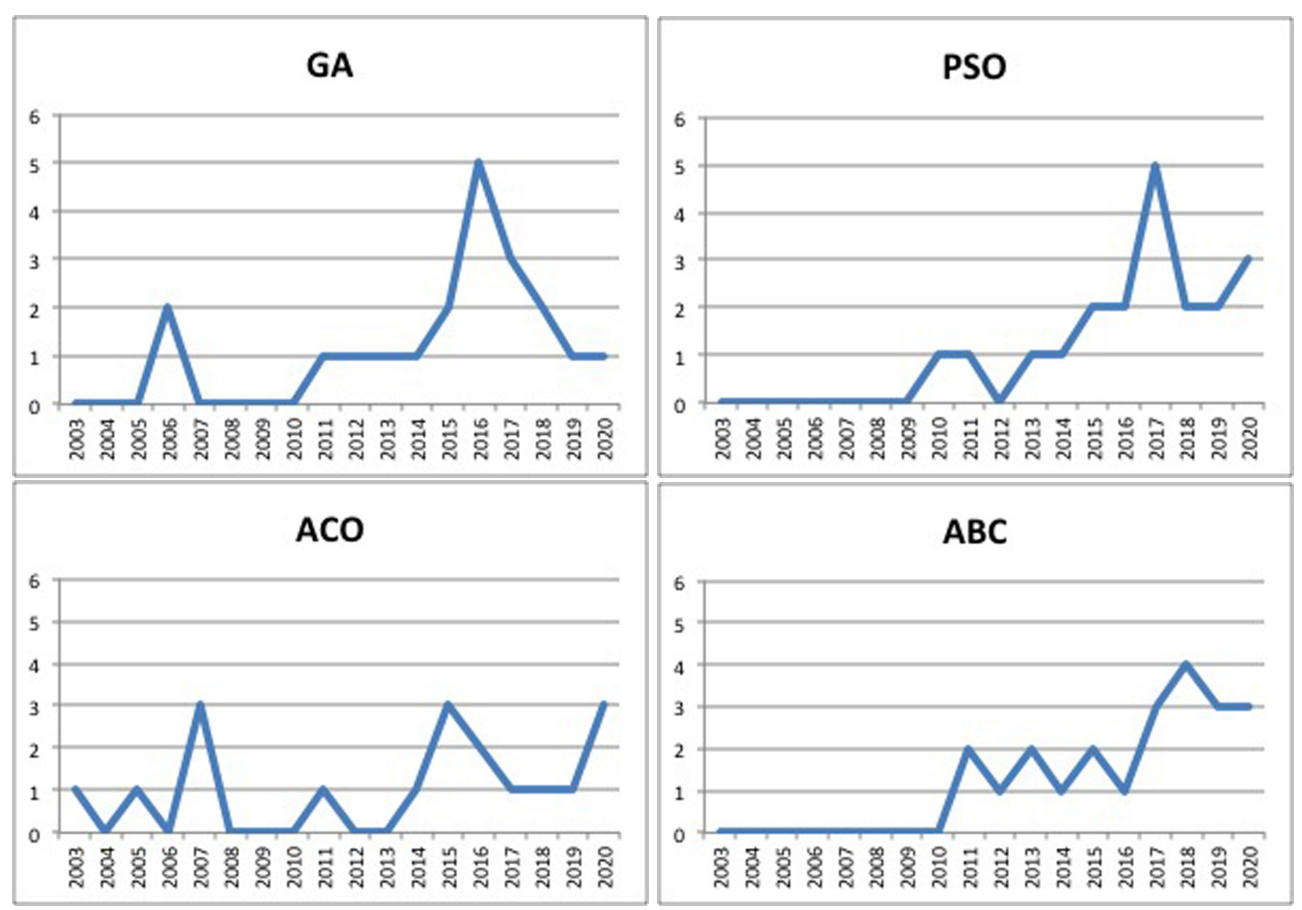

Figure 8. Yearly publication frequency of bio-inspired-techniques-based image segmentation.

\subsection{Parameter Settings}

Even though the AI algorithms solved the issue of parameter setting for the segmentation techniques, this issue arises in the implementation phase of these techniques. These parameters play an important role when working with the segmentation techniques. The four AI techniques together require setting the population size and the number of iterations/runs.

For the GA, only the crossover $(P c)$ and the mutation $(P m)$ ratios require suitable values. Table 1 summarizes the values set by the different investigated works (from 2003 to 2020). As displayed, most of the related works set $P_{c} 0.8$ and 0.9 , while $P_{m}$ was set to less than 0.05 . The population size varied between 10 and 256 , which is very difficult to recommend a value/range, because this parameter is highly dependent on the dataset. Like the population size, the iteration number varied between 20 and 3000. This parameter aims at ensuring that the obtained result is the best one and cannot be enhanced anymore. So, it clearly depends on the problem under discussion. In GA-based optimization problems, it is recommended to have a small population size and a large number of iterations [18]

PSO involves at least six parameters to set including the cognitive and social parameters (C1) and (C2), the maximum and minimum Velocity (Vmax and Vmin), and the inertia $(\omega)$. Table 2 displays the parameters' values set by the related works (from 2003 to 2020). As seen, $C 1$ and $C 2$ were set to $[0.5,2]$. The $V_{\max }$ and $V_{\min }$ were set to $[-5,5]$. The inertia was employed instead of the minimum and maximum velocity; its value varied between 0.4 and 0.9. Like the GA, the number of iterations and the population size are directly related to the tackled problem. In PSO-based optimization problems, it is recommended to take a large population with a minimum number of iterations [18].

The ACO has five parameters to set: the evaporation of the pheromone trail $(\rho)$, the initial pheromone quantity $(\tau 0)$, the maximum pheromone quantity $\left(\tau_{\max }\right)$, the influence factor-the relative importance of the residual pheromones- $(\alpha)$, and the relative importance of the pheromone trails $(\beta)$. Table 3 recapitulates these parameters' values. Most of the presented studies set the evaporation of the pheromone trail to 0.01 . The initial pheromone quantity was set between 0.001 and 1 . The maximum pheromone quantity was adjusted to 50. The influence factor and the relative importance of the pheromone trails varied between 0.1 and 5 . The parameters should be set simultaneously. It is recommended to set a low value $(<1.5)$ for $\alpha$ and $\rho$ and a high value for $\beta$ and $\tau_{\max }(20$ and 80 , respec- 
tively) [108]. The number of ants was mostly set to 10, whereas the number of iterations varied between 250 and 2500. Again, these two parameters are problem-dependent.

The $\mathrm{ABC}$ encompasses the number of bee colonies $(N P)$, the total number of food sources $(F N)$, and the number of iterations (Iter). Table 4 sums up the values of these parameters. The $N P$ varied between 15 and 50, while $F N$ was set to 20 . The number of iterations was in the range of $[20,200]$.

\subsection{Datasets}

Many datasets were investigated in the surveyed bio-inspired-based image-segmentation studies, which were obtained from different sources. They are mainly categorized into seven groups: (1) real images (the gray images such as Cameraman, Lena, RGB, etc....), (2) medical datasets, (3) Berkeley images, (4) computed tomography (CT), (5) side scan sonar, (6) the MIAS database, and (7) the other datasets category. The other datasets category includes simulated thermal images, Science, Photo Library, the CHASE database, the BSDS300 RIM-ONE dataset, the Aviris Indian Pines hyperspectral images, very-highspatial-resolution (VHSR), aerial imagery, infrared images, the DRIVE database, the STARE database, texture images, the USC-SIPI Image Database, and IBSR data banks. Figure 9 shows the number of times each category was used in the surveyed articles.

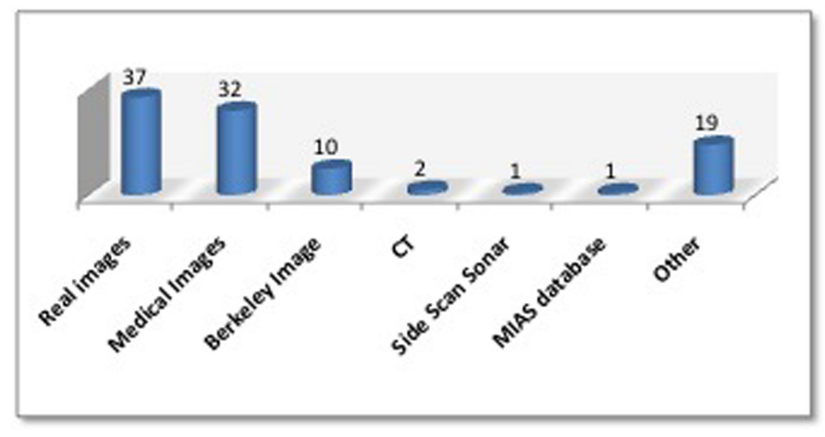

Figure 9. Categorization of image sources based on the number of times that they were used in the reviewed research articles from 2003 to 2020.

Real images (gray) that were frequently used are: Cameraman, Peppers, Lena , House, Mandrill, and Rice images. Other real images that were infrequently used are: table lamp, ceiling light, lifting body, fish man, car, wheel, airplane, satellite, hallway, foreman, container, coast guard, jet, banana, shapes, chairs, and tank.

Image segmentation plays an essential role in medical-image processing to assist in the diagnosis systems. For this reason, medical images were widely used in the surveyed bioinspired image-processing algorithms as shown in Figure 9. Medical applications include MRI brain images, heart-ventricle images, cardiac images, CT-scan images, skin-lesions demoscopic images, brain light microscopy, stomach CT, medical images from RIDER and BRATS datasets, two-dimensional electrophoresis gel images, cerebral MRI, MRI-guided prostate biopsies, noisy MRI images, and brain-tumor MRIs.

Figure 10 displays the number of times these datasets were studied with each bioinspired algorithm. It shows that real images and medical images are the most frequently used datasets for all the algorithms especially with the $\mathrm{ABC}$ algorithm. Berkeley images are also common with the $\mathrm{ABC}$ algorithm. CT was only used with the GA and PSO. The figure shows that PSO was applied to the seven dataset categories mentioned in the study. 


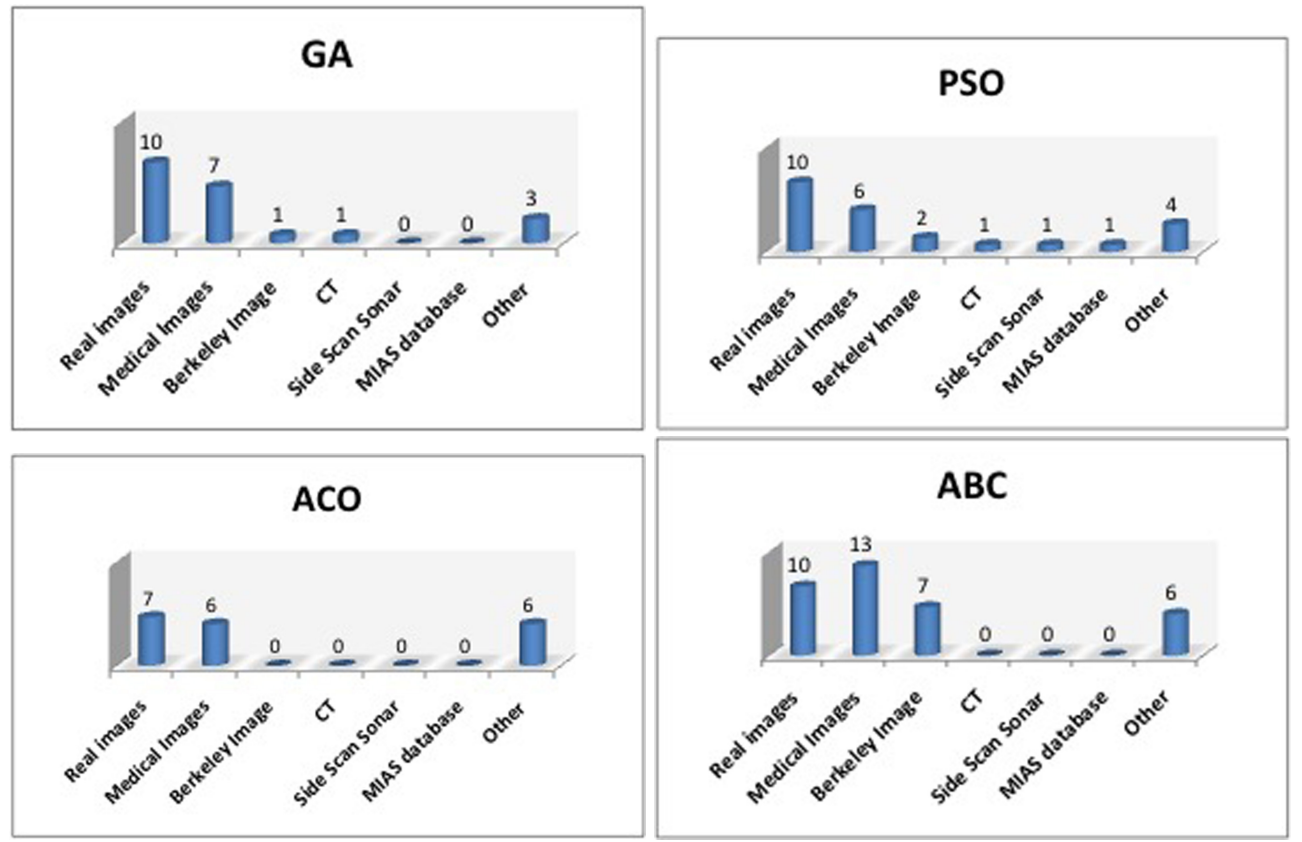

Figure 10. The frequency that each image source was studied with using each bio-inspired algorithm

\subsection{Evaluation Metrics}

Evaluation metrics are important to measure the quality of the statistical results. It is essential to use more than one evaluation metric to evaluate one model. This is because a model may produce good results using one evaluation metric but may perform weakly using another metric. There are many evaluation metrics used in the image-segmentation field, and most of them are defined below.

Accuracy: measures how close the results are to the desired value calculated as the ratio of the correct predictions to the total sample.

Error rate: is the inaccuracy of the predicted output values of the categorical dataset.

Sensitivity: is the amount of positives that are correctly recognized.

Precision: is the fraction of the related instances within the retrieved instances.

Recall: is the fraction of all the quantities of the related instances that are actually retrieved. Specificity: is the amount of negatives that are recognized correctly.

F measure: is a measure of accuracy calculated by the precision and recall of the experiment, which is also called the Dice index or the Dice similarity coefficient. It indicates no spatial overlap of the binary segmentation results when it equals zero and indicates complete overlap when it equals one.

Peak Signal-to-Noise Ratio (PSNR): is related to the ratio of the highest possible value of a signal and the value of noise that affects signal representation. In the case of image segmentation, the signal refers to the original data, and the noise refers to the error introduced by compression.

The Jaccard Similarity Coefficient (The Jaccard Index): measures the similarity and diversity of the finite sample sets calculated by dividing the size of the intersection by the size of the union.

Structural-Similarity-Index Measure: measures the perceptual similarity between two images. Probabilistic Rand Index: measures the similarity between two sets during the process of data clustering.

True Positive: is a test result where the experiment correctly predicts the positive result. True Negative: is a test result where the experiment correctly predicts the negative result. False Positive: is a test result where the experiment incorrectly predicts the positive result. False Negative: is a test result where the experiment incorrectly predicts the negative result. Variation of Information: is a simple linear expression that measures the distance between two clusters of mutual information. 
Local Consistency Error (LCE): measures the relative consistency between two different segmentation solutions using the average minimal refinement error of all pixels.

Global Consistency Error (GCE): measures the relative consistency between two different segmentation solutions using minimum cumulative refinement errors over all pixels in each coupling of clusters, then it finds the average of the result over all clusters.

Boundary Displacement Error (BDE): gauges the average displacement error of the boundary pixels and the nearest boundary pixels in the other segmentation.

Mean Square Error (MSE): is a positive value that measures the average squared difference between the estimated values and the real values: the smaller values of MSE are better.

Elapsed time: is the amount of time consumed from the beginning of an experiment to its finish.

\subsection{Tools}

To implement image segmentation, various tools and programming languages were used in the related works-for example, Python, Matlab, and Java.

\section{Conclusions and Suggestions}

This study discussed four bio-inspired algorithms to solve the problem of image segmentation. In general, there were many approaches followed by researchers when using these bio-inspired methods. One approach was to make modifications in these methodsfor example, by changing their objective functions, their search methods, or their updating strategies, which has also shown to improve the performance of the original methods. Another approach was to combine these methods with other algorithms with a main goal of improving the performance and overcoming the limitations of the individual methods. The combination was twofold. The first one combined the bio-inspired algorithms with other segmentation methods such as thresholding, edge detection, or clustering. The second combination involved combining the bio-inspired algorithms among themselves; for example, PSO was used to initialize the population of ABC in [98]. These approaches proved to improve the quality and accuracy of the results as well as to reduce the computation time. Considering these approaches, researchers can propose new techniques for image segmentation by utilizing the advantages of some methods to overcome the limitations of others.

In view of the first aforementioned combination, some segmentation techniques have not been considered for such an approach such as the split and merge approach. Moreover, the seeded region growing was only used once with ABC and once with PSO. Similarly, the Markov random model was only combined with ACO. Therefore, it is recommended to explore combining such algorithms that have not been well studied with the bio-inspired techniques and analyze their performance.

Using the bio-inspired algorithms for optimization problems has proven to be efficient as they help to avoid falling into a local minimum. This has proven its efficiency when treating image segmentation as an optimization problem. The four considered bio-inspired algorithms have been used to find the optimal parameter values needed in other techniques such as finding the optimal clusters centers without knowing the number of clusters for clustering techniques, determining the optimal threshold values in the thresholding problem, and finding the optimal coefficients for edge detection. This optimization is recommended for future work.

To sum up, some directions for future research could be suggested:

- Encourage the hybridization of the segmentation method with the bio-inspired algorithms.

- Modify the bio-inspired algorithms (combined with the segmentation methods) to improve their performance. This can be done by enhancing the movement strategy, enhancing the communication between individuals, and/or improving the evaluation process, etc. 
- Combine the bio-inspired algorithms with other well-known segmentation methods such as the split and merge approach, region growing, and the Markov random model.

- Employ a second bio-inspired algorithm to enhance the performance of the principal bio-inspired technique that will be combined with a segmentation method.

- Treat the image-segmentation problem as an optimization problem for parameter setting.

- Investigate the recent bio-inspired algorithms such as the lion optimization method, the firefly algorithm, the camel algorithm, the elephant search algorithm, and the wolf optimization algorithm.

- Investigate the use of the bio-inspired image-segmentation approaches with other datasets that have not been considered such as urban-facade image segmentation.

- Propose a new clustering technique for image segmentation including the projectionpursuit clustering method to find regions into an image.

- Compare the investigated methods to the deep-learning methods. These methods have achieved very good results, so they provide a baseline for comparison against other algorithms.

- $\quad$ Encourage research in the image-segmentation-based medical field.

- Apply the proposed techniques on video processing.

Author Contributions: Conceptualization, S.L.-M.-S. and R.A.; methodology, S.L.-M.-S.; validation, S.L.-M.-S., R.A. and S.A.; formal analysis, S.L.-M.-S., R.A. and S.A.; investigation, S.L.-M.-S., R.A. and S.A.; resources, S.L.-M.-S., R.A. and S.A.; writing-original draft preparation, S.L.-M.-S., R.A. and S.A.; writing - review and editing, S.L.-M.-S., R.A. and S.A.; visualization, R.A. All authors have read and agreed to the published version of the manuscript.

Funding: The authors would like to acknowledge the support of Prince Sultan University for paying the article processing charges (APC) of this publication.

Acknowledgments: The authors would like to acknowledge the support of Prince Sultan University for paying the article processing charges (APC) of this publication. This work was also supported by Artificial Intelligence and Data Analytics Lab (AIDA), Prince Sultan University, Riyadh, Saudi Arabia.

Conflicts of Interest: The funders had no role in the design of the study; in the collection, analyses, or interpretation of data; in the writing of the manuscript; or in the decision to publish the results.

\section{Abbreviations}

The following abbreviations are used in this manuscript:

$\begin{array}{ll}\text { AI } & \text { Artificial intelligence } \\ \text { GA } & \text { Genetic algorithm } \\ \text { PSO } & \text { Particle swarm optimization } \\ \text { ABC } & \text { Artificial bee colony optimization } \\ \text { ACO } & \text { Ant colony optimization } \\ \text { PDE } & \text { Partial differential equation } \\ \text { ANN } & \text { Artificial neural network } \\ \text { FCM } & \text { Fuzzy C-means } \\ \text { KFCM } & \text { Kernel-based fuzzy C-means } \\ \text { RGB } & \text { Red green blue } \\ \text { MRI } & \text { Magnetic resonance imaging } \\ \text { SSIM } & \text { Structural-similarity-index measure } \\ \text { PSNR } & \text { Peak signal-to-noise ratio } \\ \text { MSE } & \text { Mean square error } \\ \text { MRF } & \text { Markov random field }\end{array}$




\section{References}

1. Omarov, B.; Saparkhojayev, N.; Shekerbekova, S.; Akhmetova, O.; Sakypbekova, M.; Kamalova, G.; Alimzhanova, Z.; Tukenova, L.; Akanova, Z. Artificial Intelligence in Medicine: Real Time Electronic Stethoscope for Heart Diseases Detection. Comput. Mater. Contin. 2022, 70, 2815-2833. [CrossRef]

2. Eberhart, R.C.; Shi, Y.; Kennedy, J. Swarm Intelligence; Elsevier: Amsterdam, The Netherlands, 1995.

3. Holland, J. Adaptation in Natural and Artificial Systems; University of Michigan Press: Ann Arbor, MI, USA, 1975.

4. Karaboga, D. An Idea Based on Honey Bee Swarm for Numerical Optimization; Technical Report, Technical Report-tr06; Erciyes University, Engineering Faculty, Computer Engineering Department: Kayseri, Turkey, 2005.

5. Dorigo, M.; Gambardella, L.M. Ant colony system: A cooperative learning approach to the traveling salesman problem. IEEE Trans. Evol. Comput. 1997, 1, 53-66. [CrossRef]

6. Khan, A.; Ravi, S. Image segmentation methods: A comparative study. Int. J. Soft Comput. Eng. IJSCE 2013, 3, 84-93.

7. Sonawane, M.; Dhawale, C. A brief survey on image segmentation methods. Int. J. Comput. Appl. 2015, $975,8887$.

8. Liang, Y.; Zhang, M.; Browne, W.N. Image segmentation: A survey of methods based on evolutionary computation. In Asia-Pacific Conference on Simulated Evolution and Learning; Springer: Berlin/Heidelberg, Germany, 2014; pp. 847-859.

9. Zaitoun, N.M.; Aqel, M.J. Survey on image segmentation techniques. Procedia Comput. Sci. 2015, 65, 797-806. [CrossRef]

10. Yuheng, S.; Hao, Y. Image Segmentation Algorithms Overview. arXiv 2017, arXiv:1707.02051.

11. Chauhan, A.S.; Silakari, S.; Dixit, M. Image segmentation methods: A survey approach. In Proceedings of the IEEE 2014 Fourth International Conference on Communication Systems and Network Technologies (CSNT), Bhopal, India, 7-9 April 2014; pp. 929-933.

12. Chouhan, S.S.; Kaul, A.; Singh, U.P. Image Segmentation Using Computational Intelligence Techniques: Review. Arch. Comput. Methods Eng. 2018, 26, 533-596.

13. Chouhan, S.S.; Kaul, A.; Singh, U.P. Soft computing approaches for image segmentation: A survey. Multimed. Tools Appl. 2018, 77, 28483-28537. [CrossRef]

14. Rew, J.; Kim, H.; Hwang, E. Hybrid segmentation scheme for skin features extraction using dermoscopy images. Comput. Mater. Contin. 2021, 69, 801-817. [CrossRef]

15. Khan, K.; Khan, R.U.; Albattah, W.; Nayab, D.; Qamar, A.M.; Habib, S.; Islam, M. Crowd Counting Using End-to-End Semantic Image Segmentation. Electronics 2021, 10, 1293. [CrossRef]

16. Huang, H.Y.; Liu, Z.H. Stereo Matching with Spatiotemporal Disparity Refinement Using Simple Linear Iterative Clustering Segmentation. Electronics 2021, 10, 717. [CrossRef]

17. Hassanien, A.E.; Emary, E. Swarm Intelligence: Principles, Advances, and Applications; CRC Press: Boca Raton, FL, USA, 2016.

18. Berro, A.; Marie-Sainte, S.L.; Ruiz-Gazen, A. Genetic algorithms and particle swarm optimization for exploratory projection pursuit. Ann. Math. Artif. Intell. 2010, 60, 153-178. [CrossRef]

19. Otsu, N. A threshold selection method from gray-level histograms. IEEE Trans. Syst. Man Cybern. 1979, 9, 62-66. [CrossRef]

20. Yue, X.; Zhang, H. Modified hybrid bat algorithm with genetic crossover operation and smart inertia weight for multilevel image segmentation. Appl. Soft Comput. 2020, 90, 106157. [CrossRef]

21. Srikanth, M.V.; Prasad, V.V.K.D.V.; Prasad, K.S. An Improved Firefly Algorithm-Based 2-D Image Thresholding for Brain Image Fusion. Int. J. Cogn. Informatics Nat. Intell. (IJCINI) 2020, 14, 60-96. [CrossRef]

22. Rahaman, J.; Sing, M. An efficient multilevel thresholding based satellite image segmentation approach using a new adaptive cuckoo search algorithm. Expert Syst. Appl. 2021, 174, 114633. [CrossRef]

23. Chakraborty, F.; Durgapur, N.; India, D. Elephant Herding Optimization for Multi-Level Image Thresholding. Int. J. Appl. Metaheuristic Comput. 2020, 11, 167-176. [CrossRef]

24. Boulanouar, S.; Lamiche, C. A New Hybrid Image Segmentation Method Based on Fuzzy C-Mean and Modified Bat Algorithm. Univ. Bahrain Sci. J. 2020, 9, 677-687.

25. Hrosik, R.C.; Tuba, E.; Dolicanin, E.; Jovanovic, R.; Tuba, M. Brain Image Segmentation Based on Firefly Algorithm Combined with K-means Clustering. Stud. Inform. Control 2019, 28, 167-176. [CrossRef]

26. Wachs-Lopes, G.; Santos, R.; Saito, N.; Rodrigues, P. Recent nature-Inspired algorithms for medical image segmentation based on tsallis statistics. Commun. Nonlinear Sci. Numer. Simul. 2020, 88, 105256. [CrossRef]

27. Xu, L.; Jia, H.; Lang, C.; Peng, X.; Sun, K. A Novel Method for Multilevel Color Image Segmentation Based on Dragonfly Algorithm and Differential Evolution. IEEE Access 2019, 7, 19502-19538. [CrossRef]

28. Gonzalez, C.I.; Melin, P.; Castro, J.R.; Castillo, O.; Mendoza, O. Optimization of interval type-2 fuzzy systems for image edge detection. Appl. Soft Comput. 2016, 47, 631-643. [CrossRef]

29. Singh, V.; and, A.K.M. Cardiac image segmentation using Simulated Genetic algorithm. In Proceedings of the 2015 International Conference on Advances in Computer Engineering and Applications, Ghaziabad, India, 19-20 March 2015; pp. 1024-1027.

30. Wang, J.; Zhang, F.; Li, P. Medical image segmentation based on 2D maximum fuzzy entropy and improved genetic algorithm. In Proceedings of the 2015 8th International Congress on Image and Signal Processing (CISP), Shenyang, China, 14-16 October 2015; pp. 1583-1587.

31. Zhu, W.; Shen, Y. A segmentation approach for tissue images using non-dominated sorting GA. In Proceedings of the 2016 10 th IEEE International Conference on Anti-counterfeiting, Security, and Identification (ASID), Xiamen, China, 23-25 September 2016; pp. 1-5. [CrossRef] 
32. Lu, S.; Xu, C.; Zhong, R.Y. An active RFID tag-enabled locating approach with multipath effect elimination in AGV. IEEE Trans. Autom. Sci. Eng. 2016, 13, 1333-1342. [CrossRef]

33. Kaur, A.; Kaur, P. An integrated approach for Diabetic Retinopathy exudate segmentation by using Genetic Algorithm and Switching Median Filter. In Proceedings of the 2016 International Conference on Image, Vision and Computing (ICIVC), Xi'an, China, 26-28 July 2016; pp. 119-123.

34. Sun, G.; Zhang, A.; Yao, Y.; Wang, Z. A novel hybrid algorithm of gravitational search algorithm with genetic algorithm for multi-level thresholding. Appl. Soft Comput. 2016, 46, 703-730. [CrossRef]

35. Ghosh, P.; Mitchell, M.; Tanyi, J.A.; Hung, A.Y. Incorporating priors for medical image segmentation using a genetic algorithm. Neurocomputing 2016, 195, 181-194. [CrossRef]

36. Chandra, G.R.; Rao, K.R.H. Tumor Detection in Brain Using Genetic Algorithm. In Procedia Computer Science; Elsevier B.V.: Amsterdam, The Netherlands, 2016; Volume 79, pp. 449-457. [CrossRef]

37. Abdel-Khalek, S.; Ishak, A.B.; Omer, O.A.; Obada, A.S.F. A two-dimensional image segmentation method based on genetic algorithm and entropy. Optik 2017, 131, 414-422. [CrossRef]

38. Ishak, A.B. A two-dimensional multilevel thresholding method for image segmentation. Appl. Soft Comput. 2017, 52, 306-322. [CrossRef]

39. Singh, V.; Misra, A.K. Detection of plant leaf diseases using image segmentation and soft computing techniques. Inf. Process. Agric. 2017, 4, 41-49. [CrossRef]

40. Ashour, A.S.; Hawas, A.R.; Guo, Y.; Wahba, M.A. A novel optimized neutrosophic k-means using genetic algorithm for skin lesion detection in dermoscopy images. Signal Image Video Process. 2018, 12, 1311-1318. [CrossRef]

41. Gao, B.; Li, X.; Woo, W.L.; Tian, G.Y. Physics-based image segmentation using first order statistical properties and genetic algorithm for inductive thermography imaging. IEEE Trans. Image Process. 2018, 27, 2160-2175. [CrossRef] [PubMed]

42. Khan, M.A.; Lali, M.I.U.; Sharif, M.; Javed, K.; Aurangzeb, K.; Haider, S.I.; Altamrah, A.S.; Akram, T. An Optimized Method for Segmentation and Classification of Apple Diseases Based on Strong Correlation and Genetic Algorithm Based Feature Selection. IEEE Access 2019, 7, 46261-46277. [CrossRef]

43. Abd Elaziz, M.; Ewees, A.A.; Oliva, D. Hyper-heuristic method for multilevel thresholding image segmentation. Expert Syst. Appl. 2020, 146, 113201. [CrossRef]

44. Kanungo, P.; Nanda, P.; Samal, U. Image segmentation using thresholding and Genetic Algorithm. In Proceedings of the Conference on Soft Computing Technique for Engineering Applications, Rourkela, India, 24-26 March 2006.

45. Kanungo, P.; Nanda, P. Parallel Genetic Algorithm Based Thresholding for Image Segmentation. In Proceedings of the National Seminar on IT and Soft Computing ITSC06, Nagpur, India, 17-18 November 2006.

46. Banimelhem, O.; Yahya, A. Multi-thresholding image segmentation using Genetic Algorithm. In Proceedings of the International Conference on Image Processing, Computer Vision, and Pattern Recognition (IPCV), Las Vegas, NV, USA, 18-21 July 2011; pp. 1-6.

47. Halder, A.; Pramanik, S. An unsupervised dynamic image segmentation using fuzzy hopfield neural network based genetic algorithm. arXiv 2012, arXiv:1205.6572.

48. Hole, M.K.R.; Gulhane, V.S.; Shellokar, N.D. Application of Genetic Algorithm for Image Enhancement and Segmentation. Int. J. Adv. Res. Comput. Eng. Technol. (IJARCET) 2013, 2, 1342.

49. Dilpreet, K.; Yadwinder, K. Intelligent medical image segmentation using FCM, GA and PSO. Int. J. Comput. Sci. Inf. Technol. 2014, 5, 6089-6093.

50. Feng, Z.; Zhang, B. Fuzzy clustering image segmentation based on particle swarm optimization. Telkomnika (Telecommun. Comput. Electron. Control) 2015, 13, 128-136. [CrossRef]

51. Mekhmoukh, A.; Mokrani, K. Improved Fuzzy C-Means based Particle Swarm Optimization (PSO) initialization and outlier rejection with level set methods for MR brain image segmentation. Comput. Methods Programs Biomed. 2015, 122, 266-281. [CrossRef] [PubMed]

52. Zhao, J.; Wang, X.; Zhang, H.; Hu, J.; Jian, X. Side scan sonar image segmentation based on neutrosophic set and quantum-behaved particle swarm optimization algorithm. Mar. Geophys. Res. 2016, 37, 229-241. [CrossRef]

53. Hu, Y.; Yu, M.; Du, J. An improved image segmentation approach using FGFCM with an edges-based neighbor selection strategy and PSO. In Proceedings of the 2017 36th Chinese Control Conference (CCC), Dalian, China, 26-28 July 2017; pp. 10951-10955.

54. Na, L.; Yan, J.; Shu, L. Application of PSO algorithm with dynamic inertia weight in medical image thresholding segmentation. In Proceedings of the 2017 IEEE 19th International Conference on e-Health Networking, Applications and Services (Healthcom), Dalian, China, 12-15 October 2017; pp. 1-4. [CrossRef]

55. Taie, S.; Ghonaim, W. A New Image Segmentation Algorithm Based on Particle Swarm Optimization and Rough Set. J. Comput. 2017, 13, 130-138.

56. Suresh, S.; Lal, S. Multilevel thresholding based on Chaotic Darwinian Particle Swarm Optimization for segmentation of satellite images. Appl. Soft Comput. 2017, 55, 503-522. [CrossRef]

57. Li, Y.; Bai, X.; Jiao, L.; Xue, Y. Partitioned-cooperative quantum-behaved particle swarm optimization based on multilevel thresholding applied to medical image segmentation. Appl. Soft Comput. 2017, 56, 345-356. [CrossRef]

58. Tian, D.; Shi, Z. MPSO: Modified particle swarm optimization and its applications. Swarm Evol. Comput. 2018, 41, 49-68. [CrossRef] 
59. Gautam, K.; Singhai, R. Color Image Segmentation Using Particle Swarm Optimization in Lab Color Space. Int. J. Eng. Dev. Res. IJEDR 2018, 6, 373-377.

60. Sharif, M.; Amin, J.; Raza, M.; Mussarat, Y.; Satapathy, S. An Integrated Design of Particle Swarm Optimization (PSO) with Fusion of Features for Detection of Brain Tumor. Pattern Recognit. Lett. 2019, 129, 150-157.

61. Astuti, N.R.D.P.; Mardhia, M.M. Multilevel thresholding hyperspectral image segmentation based on independent component analysis and swarm optimization methods. Int. J. Adv. Intell. Inform. 2019, 5, 66-75. [CrossRef]

62. Sengar, S.S.; Mukhopadhyay, S. Motion segmentation-based surveillance video compression using adaptive particle swarm optimization. Neural Comput. Appl. 2020, 32, 11443-11457. [CrossRef]

63. Di Martino, F.; Sessa, S. PSO image thresholding on images compressed via fuzzy transforms. Inf. Sci. 2020, 506, 308-324. [CrossRef]

64. Farshi, T.R.; Drake, J.H.; Özcan, E. A Multimodal Particle Swarm Optimization-based Approach for Image Segmentation. Expert Syst. Appl. 2020, 149, 113233.

65. Mohsen, F.; Hadhoud, M.M.; Moustafa, K.; Ameen, K. A new image segmentation method based on particle swarm optimization. Int. Arab J. Inf. Technol. 2010, 9, 487-493.

66. Palus, H.; Bereska, D. Region-based Colour Image Segmentation. In Proceedings of the 5th Workshop Farbbildverarbeitung Ilmenau, Ilmenau, Germany, 7-8 October 1999; Volume 67, p. 74.

67. Mohsen, F.M.; Hadhoud, M.M.; Amin, K. A new optimization-based image segmentation method by particle swarm optimization. IJACSA Int. J. Adv. Comput. Sci. Appl. Spec. Issue Image Process. Anal. 2011. [CrossRef]

68. Raju, N.G.S.; Rao, P.A.N. Particle Swarm Optimization Methods for Image Segmentation Applied in Mammography. Int. J. Eng. Res. Appl. 2013, 3, 1572-1579.

69. Tandan, A.; Raja, R.; Chouhan, Y. Image Segmentation Based on Particle Swarm Optimization Technique. Int. J. Sci. Eng. Technol. Res. (IJSETR) 2014, 3, 257-260.

70. Dewan, S.; Bajaj, S.; Prakash, S. Using Ant's Colony Algorithm for improved segmentation for number plate recognition. In Proceedings of the 2015 IEEE/ACIS 14th International Conference on Computer and Information Science (ICIS), Las Vegas, NV, USA, 28 June-1 July 2015, pp. 313-318.

71. Aslam, A.; Khan, E.; Beg, M.S. Multi-Threading based Implementation of Ant-Colony Optimization Algorithm for Image Edge Detection. In Proceedings of the 2015 Annual IEEE India Conference (INDICON), New Delhi, India, 17-20 December 2015; Volume 151, pp. 10-17.

72. Ye, Z.; Wang, M.; Jin, H.; Liu, W.; Lai, X. An Image Thresholding Approach Based on Ant Colony Optimization Algorithm Combined with Genetic Algorithm. Int. J. Intell. Syst. Appl. 2015, 7, 8-15. [CrossRef]

73. Arnay, R.; Fumero, F.; Sigut, J. Ant Colony Optimization-based method for optic cup segmentation in retinal images. Appl. Soft Comput. J. 2016, 52, 409-417.

74. Zou, G. Ant colony clustering algorithm and improved markov random fusion algorithm in image segmentation of brain images. Int. J. Bioautom. 2016, 20, 505; Technical Report 4.

75. Selmani, A.; Seddik, H.; Braiek, E.B. A novel ant colonies approach to medical image segmentation. In Proceedings of the 2017 IEEE 14th International Multi-Conference on Systems, Signals \& Devices (SSD), Marrakech, Morocco, 28-31 March 2017; pp. 22-26.

76. Al-Ruzouq, R.; Shanableh, A.; Gibril, M.B.A.; Al-Mansoori, S. Image segmentation parameter selection and ant colony optimization for date palm tree detection and mapping from very-high-spatial-resolution aerial imagery. Remote Sens. 2018, 10, 1413. [CrossRef]

77. Singh, R.; Vashishath, M.; Kumar, S. Ant colony optimization technique for edge detection using fuzzy triangular membership function. Int. J. Syst. Assur. Eng. Manag. 2019, 10, 91-96. [CrossRef]

78. Khudov, H.; Ruban, I.; Makoveichuk, O.; Pevtsov, H.; Khudov, V.; Khizhnyak, I.; Fryz, S.; Podlipaiev, V.; Polonskyi, Y.; Khudov, R. Development of methods for determining the contours of objects for a complex structured color image based on the ant colony optimization algorithm. EUREKA Phys. Eng. 2020, 2020, 34-47. [CrossRef]

79. Devarajan, D.; Ramesh, S.M.; Gomathy, B. A metaheuristic segmentation framework for detection of retinal disorders from fundus images using a hybrid ant colony optimization. Soft Comput. 2020, 24, 13347-13356. [CrossRef]

80. Sandhya, G.; Kande, G.B.; Savithri, T.S. Q4: Segmentation of Magnetic Resonance Brain Images Using the Advanced Ant Colony Optimization Technique. J. Biomim. Biomater. Biomed. Eng. 2020, 44, 37-49. [CrossRef]

81. Gonzalez, R.C.; Woods, R.E. Digital Image Processing; Prentice Hall: Harlow, UK, 2007.

82. Maini, R.; Sohal, J. Performance evaluation of Prewitt edge detector for noisy images. GVIP J. 2006, 6, 39-46.

83. Roberts, L.G. Machine Perception of Three Dimensional Solids. In Optical and Electro-Optical Information Processing; MIT Press: Cambridge, MA, USA, 1965; pp. 159-197.

84. Ouadfel, S.; Batouche, M. MRF based image segmentation using Ant Colony System. ELCVIA Electron. Lett. Comput. Vis. Image Anal. 2003, 2, 12-24. [CrossRef]

85. Ouadfel, S.; Batouche, M.; Talhi, S. Ant Colonies For MRF Based Image Segmentation. In Proceedings of the 3rd International Conference: Sciences of Electronic, Technologies of Information and Telecommunications, Sousse, Tunisia, 27-31 March 2005.

86. Zhao, B.; Zhu, Z.; Mao, E.; Song, Z. Image segmentation based on ant colony optimization and K-means clustering. In Proceedings of the 2007 IEEE International Conference on Automation and Logistics, Jinan, China, 18-21 August 2007, pp. 18-21. 
87. Laptik, R.; Navakauskas, D. Application of ant colony optimization for image segmentation. Electron. Electr. Eng. J. 2007, 80, 13-18.

88. Han, Y.; Shi, P. An improved ant colony algorithm for fuzzy clustering in image segmentation. Neurocomput. J. 2007, 70, 665-671. [CrossRef]

89. Feng, Y.; Wang, Z. Ant Colony Optimization for Image Segmentation. In Ant Colony Optimization; Ostfeld, A., Ed.; IntechOpen: Rijeka, Croatia, 2011; Chapter 17.

90. Xumin, L.; Shi, N.; Cailing, L. Image segmentation algorithm based on improved ant colony algorithm. Int. J. Signal Process. Image Process. Pattern Recognit. 2014, 7, 433-442.

91. Bouaziz, A.; Draa, A.; Chikhi, S. Artificial bees for multilevel thresholding of iris images. Swarm Evol. Comput. 2015, 21, 32-40. [CrossRef]

92. Sağ, T.; Çunkaş, M. Color image segmentation based on multiobjective artificial bee colony optimization. Appl. Soft Comput. 2015, 34, 389-401.

93. Bose, A. Fuzzy-based artificial bee colony optimization for gray image. Signal Image Video Process. 2016, 10, 1089-1096.

94. Li, L.; Sun, L.; Guo, J.; Han, C.; Zhou, J.; Li, S. A quick artificial bee colony algorithm for image thresholding. Information $2017,8,16$. [CrossRef]

95. Huo, F.; Liu, Y.; Wang, D.; Sun, B. Bloch quantum artificial bee colony algorithm and its application in image threshold segmentation. Signal Image Video Process. 2017, 11, 1585-1592. [CrossRef]

96. Fu, Z.; Liu, Y.; Hu, H.; Wu, D.; Gao, H. An efficient method of white blood cells detection based on artificial bee colony algorithm. In Proceedings of the 2017 IEEE 29th Chinese Control And Decision Conference (CCDC), Chongqing, China, 28-30 May 2017; pp. 3266-3271.

97. Feng, Y.; Yin, H.; Lu, H.; Cao, L.; Bai, J. FCM-based quantum artificial bee colony algorithm for image segmentation. In Proceedings of the 10th International Conference on Internet Multimedia Computing and Service, Nanjing, China, 17-19 August 2018; p. 6.

98. Zhang, S.; Jiang, W.; Satoh, S. Multilevel thresholding color image segmentation using a modified artificial bee colony algorithm. IEICE Trans. Inf. Syst. 2018, E101D, 2064-2071. [CrossRef]

99. Lingappa, H.K.; Suresh, H.N.; Manvi, S.K. Medical image segmentation based on extreme learning machine algorithm in Kernel Fuzzy C-Means using artificial bee colony method. Int. J. Intell. Eng. Syst. 2018, 11, 128-136. [CrossRef]

100. Banharnsakun, A. Artificial bee colony algorithm for enhancing image edge detection. Evol. Syst. 2018, 10, 679-687.

101. Ma, L.; Wang, X.; Shen, H.; Huang, M.; Ma, L.; Wang, X.; Shen, H.; Huang, M. A novel artificial bee colony optimiser with dynamic population size for multi-level threshold image segmentation. Int. J. Bio-Inspired Comput. 2019, 13, 32-44; Technical Report 1. [CrossRef]

102. Gupta, S.; Deep, K. Hybrid sine cosine artificial bee colony algorithm for global optimization and image segmentation. Neural Comput. Appl. 2019, 6, 9521-9543.

103. Huo, F.; Sun, X. Multilevel image threshold segmentation using an improved Bloch quantum artificial bee colony algorithm. Multimed. Tools Appl. 2020, 79, 2447-2471. [CrossRef]

104. Hancer, E. Artificial Bee Colony: Theory, Literature Review, and Application in Image Segmentation. In Recent Advances on Memetic Algorithms and its Applications in Image Processing. Studies in Computational Intelligence; Springer: Singapore, 2020; Volume 873, pp. 47-67. [CrossRef]

105. Alrosan, A.; Alomoush, W.; Norwawi, N.; Alswaitti, M.; Makhadmeh, S.N. An improved artificial bee colony algorithm based on mean best-guided approach for continuous optimization problems and real brain MRI images segmentation. Neural Comput. Appl. 2020, 33, 1671-1697.

106. Zhi, H.; Liu, S. Gray image segmentation based on fuzzy c-means and artificial bee colony optimization. J. Intell. Fuzzy Syst. 2020, 38, 3647-3655. [CrossRef]

107. Pare, S.; Kumar, A.; Singh, G.K.; Bajaj, V. Image Segmentation Using Multilevel Thresholding: A Research Review. Iran. J. Sci. Technol.-Trans. Electr. Eng. 2020, 44, 1-29. [CrossRef]

108. Peng, L.; Hua, Z. Parameter Selection for Ant Colony Algorithm Based on Bacterial Foraging Algorithm. Math. Probl. Eng. 2016, 2016, 6469721. [CrossRef] 